\title{
Determinación de cadmio en suelos agrícolas del cantón Pallatanga, provincia de Chimborazo, Ecuador
}

\section{Determination of cadmium in agricultural soils of Pallatanga canton, province of Chimborazo, Ecuador}

\section{Julio César Pilco Yungan ${ }^{1 *} \&$ David Eloy García Asencio²}

\author{
${ }^{1}$ Egresado de la Facultad de Ciencias Naturales, Universidad de Guayaquil. \\ ${ }^{2}$ Docente investigador de la Facultad de Ciencias Naturales, Universidad de Guayaquil, \\ Av. Raúl Gómez Lince s/n y Av. Juan Tanca Marengo, Guayaquil, Ecuador.
}

Recibido de abril 2020; recibido en forma revisada 26 de mayo 2020, aceptado 1 de junio 2020 Disponible en línea 26 de junio 2020

\begin{abstract}
Resumen
Esta investigación tuvo la finalidad de determinar la presencia de cadmio en suelos agrícolas, para lo cual se seleccionaron 6 estaciones de muestreo en dos sistemas de cultivo que se encontraron ubicados en tres pisos altitudinales. Aplicando el método de espectrofotometría de absorción atómica a llama se realizó la cuantificación de cadmio, y para la caracterización del suelo se utilizó los siguientes métodos: tablas de Munsell, Feel, AS-05, gravimetría de volatilización indirecta; y adicionalmente se emplearon dos multiparamétricos para determinar el $\mathrm{pH}$ y la conductividad eléctrica. Se demostró que la variabilidad del $\mathrm{Cd}$ difiere con los sistemas de cultivo y los pisos altitudinales con valores entre $0,5 \mathrm{mg} / \mathrm{kg}$ a $1,0 \mathrm{mg} / \mathrm{kg}$. Siendo el $94 \%$ de las muestras quienes superaron el límite máximo permisible nacional, en donde además la correlación del cadmio con las propiedades fisicoquímicas del suelo presentó una afinidad con el pH y la materia orgánica.
\end{abstract}

Palabras clave: Suelo, cadmio, agricultura, piso altitudinal.

\begin{abstract}
This investigation had the purpose of determining the presence of cadmium in agricultural soils, for which 6 sampling stations were selected in two cultivation systems that were found located in three altitudinal floors. Cadmium quantification was performed using the atomic absorption spectrophotometry to flame method, and the following methods were used to characterize the soil: Munsell tables, Feel, AS05 , indirect volatilization gravimetry; and additionally, two multiparametric were used to determine $\mathrm{pH}$ and electrical conductivity. $\mathrm{Cd}$ variability was shown to differ with culture systems and altitudinal floors with values between $0,5 \mathrm{mg} / \mathrm{kg}$ to $1,0 \mathrm{mg} / \mathrm{kg}$. Being $94 \%$ of the samples who exceeded the national maximum permissible limit, where in addition the correlation of cadmium with the physicochemical properties of the soil had an affinity with $\mathrm{pH}$ and organic matter.
\end{abstract}

Keywords: Soil, cadmium, agriculture, altitudinal floor.

\section{Introducción}

Los metales pesados son contaminantes ambientalmente importantes por tener un orden cíclico: industria, atmósfera, suelo, agua, alimentos y humano (Rodrigues, Reis, \& Lupino, 2016). Se incorporan en el cuerpo humano a través de la dieta diaria u otra forma de adición, acumulándose principalmente en los riñones, las glándulas suprarrenales, el hígado, los pulmones, el cabello y la piel, causando presión arterial alta, cáncer y daño cerebral, esto sucede cuando se supera la 
Rev. Cient. Cien. Nat. Ambien. 14(1):151-172 Junio 2020
Pilco \& García - Determinación de cadmio en suelos agrícolas del cantón Pallatanga, provincia de Chimborazo, Ecuador ingesta diaria recomendada, por ejemplo, para el contaminante cadmio se considera una ingesta segura de 15 a $50 \mu \mathrm{g} /$ día para adultos, y de 2 a $25 \mu \mathrm{g}$ /día para niños (Neelam, Vinita, \& Sibi, 2018).

Alloway \& Steinnes (1999) indicaron que el aporte de cadmio en la agricultura se da a través del agua de riego e insumos agrícolas como abonos, fertilizantes y enmiendas del suelo. Es así, que gran parte del Cd presente en el suelo se libera al ambiente a través de los cultivos, sin embargo, otras vías potenciales de pérdida de este metal incluyen la erosión, bioturbación y lixiviación. La entrada y salida de Cd es generalmente pequeña en comparación con la cantidad total que se encuentra en el sustrato y cuyas concentraciones aumentan con el tiempo siempre y cuando las adiciones sean mayores que las pérdidas (Sheppard, Grant, Sheppard, de Jong, \& Long, 2009) including soil, but agriculture uses some materials that have increased TE concentrations. Some TES (e.g., Cu, Se, and Zn.

El incremento de cadmio en los suelos agrícolas se debe a la malas prácticas como el uso excesivo de fertilizantes químicos, ya que estos a menudo contienen un porcentaje muy elevado del contaminante por su origen de roca fosfatada (Qian et al., 2016). Además, no es común que se realicen análisis de los suelos para determinar la deficiencia exacta de nutrientes que poseen, la cual es suplida por los fertilizantes químicos provocando un excesivo uso de estos por parte de los agricultores (KabataPendias, 2010).

Gran parte del Cd absorbido por la planta se retiene en la raíz, pero una parte se transfiere a la porción aérea, también se transportan cantidades variables a la semilla. El porcentaje de este metal en los cultivos depende del genotipo de la planta, que difieren entre especies, como consecuencia de las prácticas de manejo del suelo y la manipulación genética (Singh \& McLaughlin, 1999).

Una vez que el cadmio ingresa al cuerpo humano, se acumula principalmente en los órganos diana, en donde puede permanecer de 15 a 20 años y cuyos efectos se manifiestan principalmente en las personas mayores a 50 años (Alloway, 2013). Esto podría conllevar a algún tipo de cáncer o enfermedades afines como la erosión del tracto gastrointestinal, daño pulmonar, hepático o renal. La exposición crónica, además, genera un efecto depresivo sobre los niveles de noradrenalina, serotonina y acetilcolina (Danielyan \& Chailyan, 2019; Singhal, Merali, \& Hrdina, 1976).

Schroeder \& Balassa (1963), realizaron las primeras investigaciones acerca de la incorporación del Cd en la cadena alimentaria humana, a través de impurezas de este contaminante en fertilizantes y enmiendas aplicadas a los suelos agrícolas. El interés por el ciclo del Cd se incrementó a causa de la contaminación industrial de las tierras agrícolas en Japón, lo cual fue provocado por aguas residuales de la minería contaminadas con este metal, lo que condujo a grandes pérdidas humanas causadas por la enfermedad denominada "itai-itai", documentada en los años setenta (Kobayashi, 1978).

Grant, Buckley, Bailey, \& Selles (1998), determinaron que la concentración de Cd en los suelos de Canadá había aumentado con el tiempo debido a las adiciones de fertilizantes de $\mathrm{N}, \mathrm{Py} \mathrm{KCl}$, así como la incorporación de enmiendas del suelo. Además, se estimó que gran parte del Cd absorbido por la planta se retiene en la raíz, pero una parte se transfiere a la parte aérea.

Mientras tanto, en Chile, ensayos realizados por Bonomelli, Bonilla, \& Valenzuela (2003), acerca del efecto de la fertilización fosforada sobre el contenido de cadmio en cuatro tipos de suelo; alfisol, inceptisol, ultisol y andisol, dieron como resultado después de 90 días de incubación los siguientes valores; para el suelo de orden alfisol, el testigo dio un valor de; $0,021 \pm 0,002 \mathrm{a} \mathrm{mg} / \mathrm{kg}$ de $\mathrm{Cd}$, con la fertilización fosforada $0,041 \pm 0,005 \mathrm{~b} \mathrm{mg} / \mathrm{kg}$ de $\mathrm{Cd}$, para el tipo inceptisol; testigo $0,110 \pm 0,008 \mathrm{a} \mathrm{mg} / \mathrm{kg}$ de $\mathrm{Cd}$, con la fertilización fosforada $0,128 \pm 0,007 b$, para ultisol; testigo $0,010 \pm 0,0009 \mathrm{a} \mathrm{mg} / \mathrm{kg}$ de $\mathrm{Cd}$, con la fertilización fosforada $0,033 \pm 0,006 \mathrm{~b}$ y andisol; testigo $0,029 \pm 0,001 \mathrm{a} \mathrm{mg} / \mathrm{kg}$ de $\mathrm{Cd}$, con fertilización fosforada $0,081 \pm 0,005 \mathrm{~b} \mathrm{mg} / \mathrm{kg}$ de $\mathrm{Cd}$.

Con estos resultados los autores determinaron que el Cd liberado en el primer día incubación por parte del fertilizante, permaneció disponible hasta culminar las mediciones, concluyendo que los suelos necesitados de nutrientes presentan una capacidad de retención de fósforo muy alta, al mismo tiempo se incorpora Cd al sustrato, sin embargo la disponibilidad del Cd puede o no disminuir a través del tiempo, dependiendo de las características del suelo como $\mathrm{pH}$, materia orgánica y cantidad de fertilizante aplicado (Bonomelli et al., 2003).

En Ecuador se han realizado estudios sobre cadmio en hojas y pulpa de tomate, evidenciando que la mayor concentración se encuentra en la pulpa, con 0,0349 $\pm 0,0155 \mathrm{mg} / \mathrm{kg}$ de $\mathrm{Cd}$, valor que sobrepasa el límite máximo permisible establecido por la Unión Europea de 0,02 mg/kg de Cd (Escobar, 2016; UE, 2019). Por otra parte, una investigación hecha sobre toxicidad en la germinación del fréjol a diferentes concentraciones de $\mathrm{Cd}$, concluyó que la presencia de este metal afecta la productividad de este cultivo, en donde se recomienda que el suelo agrícola sea adecuado de tal manera que la concentración de Cd sea inexistente (Añazco, 2019).

Chicón (2006), estableció que los metales pesados tienden a tener mayor movilidad en un $\mathrm{pH}$ ácido, provocando el incremento de toxicidad para el cultivo. También afirma que los suelos que presentan un porcentaje mayor al $5 \%$ de materia orgánica, ostentan un nivel relativamente bajo de retención de metales por el cultivo, a consecuencia del aumento de las reacciones complejas por parte de las moléculas orgánicas. 
En cuando a la biodisponibilidad de este contaminante y sus formas en el suelo, Cargua (2010), examinó el Cd fácilmente extraíble, reducible, oxidable y residual, de 40 fincas del litoral ecuatoriano, en donde el valor medio de las formas disponibles examinadas fue el siguiente: para la provincia de El Oro 1,2 mg/ $\mathrm{kg}$, Esmeraldas 2,0 mg/kg, Guayas $1,3 \mathrm{mg} / \mathrm{kg}$, Santa Elena $1,9 \mathrm{mg} / \mathrm{kg}$, Los Ríos $0,8 \mathrm{mg} / \mathrm{kg}$ y para Azuay 1,2 $\mathrm{mg} / \mathrm{kg}$. Según el autor, estos resultados no presentan problemas con el contenido de $\mathrm{Cd}$ de la fracción fácilmente extraíble del sustrato.

Además, las características fisicoquímicas de las muestras presentaron un $\mathrm{pH}$ promedio de 6,7 , siendo este un valor neutro que predomina en suelos de tipo franco arcilloso y franco limoso, con rangos de materia orgánica que variaron de $0,6 \%$ a $10,7 \%$. Los suelos de tipo arcilloso capturan el $\mathrm{Cd}$ en el suelo, lo que contribuye a una menor acumulación de metales pesados en la planta. A su vez, la materia orgánica reacciona con este metal formando quelatos con complejos de cambio (Cargua, 2010).

Del mismo modo, en Nueva Zelanda se registró una acumulación de $\mathrm{Cd}$, en tres tipos de suelo con características distintas (alofánico ortico, ortico gley, y alofánico impedido) con el mismo historial de fertilizantes fosfatados, en donde la concentración de $\mathrm{Cd}$ en el horizonte $\mathrm{A}$ de cada suelo fue de 7 a 8 veces mayor que los horizontes más profundos. Mientras, que la concentración media de $\mathrm{Cd}$ en las muestras de la capa superior del suelo $(0-7.5 \mathrm{~cm})$ fue de $0.77 \mathrm{mg} /$ $\mathrm{kg}$, en donde no se encontró diferencias significativas en la concentración de Cd entre los tres tipos de suelo (Salmanzadeh, Balks, Hartland, \& Schipper, 2016).

Mientras tanto, en China se modificaron los estándares de contaminación del suelo a otros más rigurosos (GB15618-2018, efectivo el 1 de agosto de 2018) a consecuencia de la severa contaminación del centro y suroeste del país. Se estimó que el 19,4\% de las muestras de suelo excedieron los límites permisibles establecidos por el gobierno chino en 1995, de todas las muestras contaminadas los metales pesados y los metaloides representaron la mayoría de los contaminantes $(82,4 \%)$, y el resto $(17,6 \%)$ son contaminantes orgánicos. El contaminante Cd clasificó con el porcentaje más alto presente en los suelos agrícolas con un 7\% a comparación de los demás contaminantes estudiados (Wang, Chen, Kopittke, \& Zhao, 2019).

Así mismo, en Bangladesh se realizó una cuantificación de metales pesados, en donde a partir de los resultados se determinó que la concentración de metales en el suelo era inferior a los estándares internacionales por cual se rigió el estudio, excepto para el Cd y Cu. En donde el Cd presentó valores entre 0,19 mg/kg y 9,04 $\mathrm{mg} / \mathrm{kg}$, registrándose por encima del límite permitido establecido por el estándar holandés $(0,80 \mathrm{mg} / \mathrm{kg})$, las pautas canadienses $(1,4 \mathrm{mg} / \mathrm{kg})$ y las pautas australianas (3,0 mg/kg) (Proshad et al., 2019).
Por otra parte, según Tóth, Hermann, Da Silva, \& Montanarella (2016), la mayoría de las tierras de cultivo de la Unión Europea se pueden considerar seguras para la producción de alimento ya que se encontró un porcentaje muy bajo de metales pesados que sobrepasaron el umbral de evaluación (6.24 \%). Para el caso del Cd la mayor parte de las muestras $(72,6 \%)$ no mostraron concentraciones detectables de $\mathrm{Cd}$ y sólo el $5,5 \%$ de las muestras tienen concentraciones superiores al valor umbral de $1.0 \mathrm{mg} / \mathrm{kg}$ establecido por el ministerio del ambiente de Finlandia, además, se estableció que las regiones con mayores concentraciones medias de cadmio se pueden encontrar en Irlanda y Grecia.

Algo parecido sucedió con los resultados encontrados de 83 muestras de suelo cultivable $(0-20 \mathrm{~cm})$ de las áreas agrícolas y de pastoreo de la cuenca del río Sinú en Colombia, cuyo valor medio de Cd fue de 0.040 $\mathrm{mg} / \mathrm{kg}$, lo cual es inferior a la media de la corteza $(0,1 \mathrm{mg} / \mathrm{kg})$ y el promedio mundial $(0,41 \mathrm{mg} / \mathrm{kg})$. Sin embargo, los demás metales pesados estudiados $(\mathrm{Cu}$, $\mathrm{Ni}, \mathrm{Pb}, \mathrm{Hg}$ ) sobrepasaron los valores de referencia (Marrugo, Pinedo, \& Díez, 2017).

En los suelos de cultivo de cacao del sur del país según Chavez et al. (2015) las concentraciones de Cd y las propiedades del suelo difirieren para distintas profundidades, es decir que este metal se acumula sustancialmente en los suelos superficiales $(0-15 \mathrm{~cm})$ en donde se encontró que la provincia del Guayas las concentraciones variaron entre 0,66 a 2,59 mg/ $\mathrm{kg}$ de $\mathrm{Cd}$, probablemente debido a actividades antropogénicas (por ejemplo, riego de agua rica en Cd). Además, el contenido de $\mathrm{Cd}$ total recuperable y extraíble, según lo estimado por $\mathrm{M} 3$ Método $\mathrm{HCl}$, disminuye con la profundidad del suelo.

De igual manera, Muñoz (2017) determinó la concentración total y biodisponible del $\mathrm{Cd}$ en dos cantones de la provincia del Guayas, encontrando concentraciones inferiores al límite máximo permisible de $0,5 \mathrm{mg} / \mathrm{kg}$ hasta $2,59 \pm 0,30 \mathrm{mg} / \mathrm{Kg}$. Mientras, que para la biodisponibilidad del $\mathrm{Cd}$ de las estaciones de muestreo presentaron porcentajes mayores al 50\% e incluso en una estación se llegó al 100\%.

Escobar (2016), indicó la presencia de cadmio en los suelos de la parroquia El Quinche, ubicada en la provincia de Pichincha a 2600 m.s.n.m., para el cultivo de tomate, cuyo valor medio fue de $0,56 \mathrm{mg} / \mathrm{kg}$, lo cual no sobrepasa el límite máximo permisible establecido por la normativa ecuatoriana para el criterio de remediación de suelo de $2,0 \mathrm{mg} / \mathrm{kg}$. Sin embargo, el valor encontrado $(0,56 \mathrm{mg} / \mathrm{kg})$ supera el valor ambiental representativo para el criterio de calidad de suelo de 0,50 mg/kg establecido por el MAE (2015).

En la provincia de Chimborazo se han registrado estudios de $\mathrm{Cd}$ en tubérculos, hojas, bulbos, frutos de hortalizas e incluso en leguminosas, entre ellas el fréjol, que presentó valores mayores a 0,2 mg/kg, duplicando el límite máximo permitido por el Codex 
Alimentarius de la UE (FAO y WHO, 2019) vigente en la actualidad. En Guano se identificó Cd en papa, cebolla y brócoli con valores que oscilaron entre 0,16 y $0,21 \mathrm{mg} / \mathrm{kg}$, estos resultados no sobrepasan los valores máximos establecidos por la misma normativa (Felicita, 2018).

En el cantón Pallatanga, se ha determinado que el factor antrópico representa un 55\% del deterioro del suelo; debido al manejo indiscriminado de agroquímicos y fertilizantes, los cuales afectan la calidad y propiedades de esté. Además, el uso de agua contaminada expresa un $35 \%$ de pérdida de suelo y cultivos (Gobierno Autónomo Descentralizado Municipal del Cantón Pallatanga, 2018).

Por lo expuesto anteriormente, se consideró determinar la presencia y concentración de $\mathrm{Cd}$ en suelos agrícolas del cantón Pallatanga, provincia de Chimborazo. Dicho cantón abastece al mercado nacional con una amplia variedad de cultivos como: pimiento, pepino dulce, papas, maíz, mora, frutilla, etc., y destaca por su mayor producción de tomate y fréjol, estos dos cultivos se localizan en los primeros tres pisos altitudinales del cantón.

\section{Metodología}

\section{Área de estudio}

la presente investigación se llevó a cabo en el cantón Pallatanga, ubicado al suroeste de la Provincia de Chimborazo, a $75 \mathrm{Km}$ de Riobamba y $140 \mathrm{~km}$ de la ciudad de Guayaquil, en el occidente de Ecuador. Su superficie es de $377 \mathrm{~km}^{2}$ y se caracteriza por ser una zona montañosa con amplios valles, cuyas peculiaridades climáticas favorecen a la producción agrícola como la principal fuente económica para la localidad.

Dentro del área de estudio se seleccionaron 6 estaciones de muestreo ubicadas dentro de los tres primeros pisos altitudinales del cantón; de esas estaciones se extrajeron muestras de suelo de dos tipos de cultivo: tomate y fréjol. Según el MAE (2012), para la cordillera occidental el piso altitudinal pie montano se encuentra entre los 300 a 1300 ms.n.m., el montano bajo entre 1300 a 1900 ms.n.m., y el montano de 1900 a 2800 ms.n.m. (Tabla 1; fig. 1).

\section{Metodología de campo}

Se realizó un muestreo probabilista del tipo aleatorio simple con una periodicidad mensual, entre los meses de noviembre y diciembre del 2019, en dos sistemas de cultivos (tomate y fréjol), en tres pisos altitudinales, con un total de 36 muestras.

Una vez seleccionado las estaciones de muestreo, dos para cada piso altitudinal (Fig. 1), se prosiguió con un muestreo simple el cual consistió en extraer 3 réplicas por cada punto, con una profundidad de 0-15 $\mathrm{cm}$ según la guía técnica para el muestreo de suelo (Mendoza \& Espinoza, 2017).

El diseño experimental que se aplicó para la colecta de muestras de suelo agrícola fue el de transeptos lineales, tomando como referencia el centro del

\section{MAPA DE LAS ESTACIONES DE MUESTREO SELECCIONADOS PARA LA RECOLECCIÓN DE MUESTRAS EN PALLATANGA}

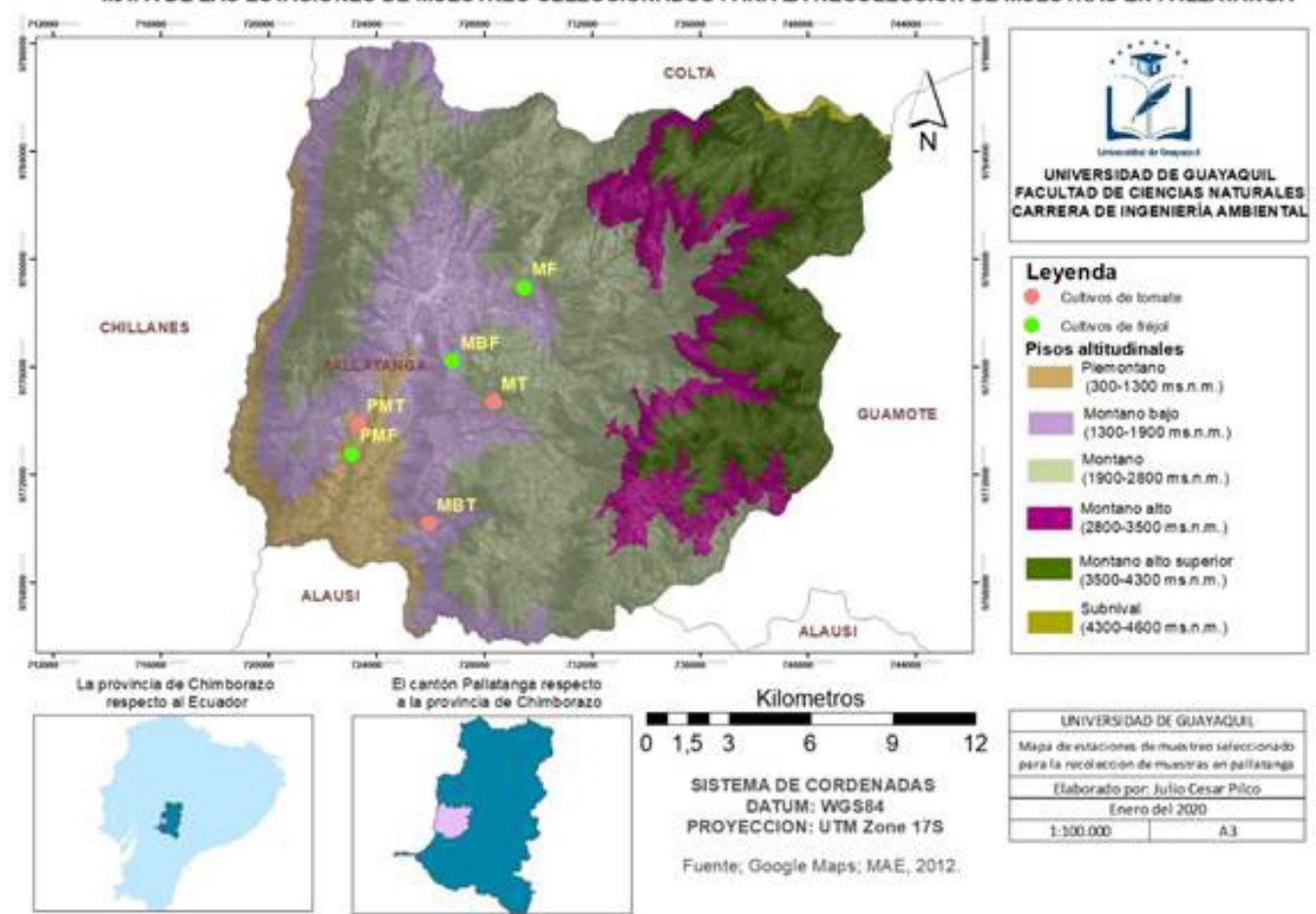

Figura 1. Área de estudio. Estaciones de muestreo seleccionadas. 
Rev. Cient. Cien. Nat. Ambien. 14(1):151-172 Junio 2020
Pilco \& García - Determinación de cadmio en suelos agrícolas del cantón Pallatanga, provincia de Chimborazo, Ecuador

Tabla 1. Datos generales de las estaciones de muestreo.

\begin{tabular}{cllccc}
\hline \multirow{2}{*}{$\begin{array}{c}\text { Piso } \\
\text { altitudinal }\end{array}$} & Cultivo & Abreviatura & Altitud & \multicolumn{2}{c}{$\begin{array}{c}\text { Coordenadas } \\
\text { UTM WG.n.m. }\end{array}$} \\
\hline \multirow{2}{*}{$\begin{array}{c}\text { Pie } \\
\text { montano }\end{array}$} & Tomate & PMT & 1289 & 723328,81 & 9773876,73 \\
\cline { 2 - 6 } & Fréjol & PMF & 1259 & 723084,71 & 9772756,79 \\
\hline \multirow{2}{*}{ Montano bajo botitud } & Tomate & MBT & 1454 & 725957,87 & 9770201,56 \\
\cline { 2 - 6 } & Fréjol & MBF & 1793 & 726833,56 & 9776216,54 \\
\hline \multirow{2}{*}{ Montano } & Tomate & MT & 2179 & 728332,63 & 9774702,88 \\
\cline { 2 - 6 } & Fréjol & MF & 2188 & 729481,25 & 9778941,51 \\
\hline
\end{tabular}

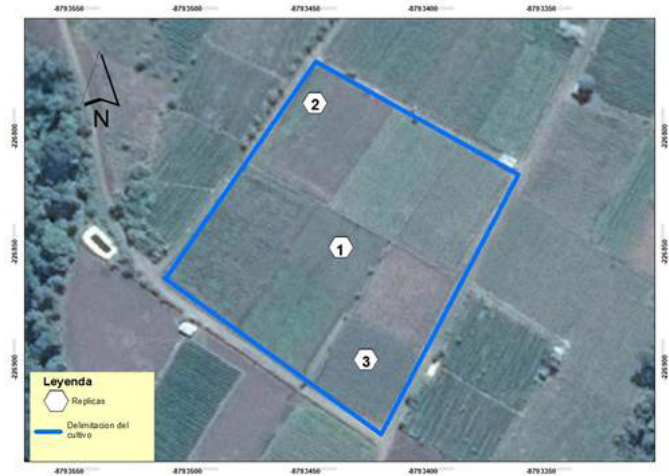

Figura 2. Diseño experimental para obtención de muestras agrícolas en cultivos de tomate y fréjol en tres pisos altitudinales del cantón Pallatanga.

cultivo como la primera réplica, y los extremos representaban la segunda y tercera réplica con la finalidad de cubrir la totalidad del cultivo (Fig. 2; anexo 1).
Para la recolección de las muestras de suelo se empleó una pala, la misma que fue esterilizada con agua destilada para evitar cualquier tipo de alteración de las muestras. Se colectaron aproximadamente $500 \mathrm{~g}$ de suelo en fundas ziploc, las mismas que fueron rotuladas in situ para su posterior análisis en laboratorio (Fig. 3).

Metodología de laboratorio

\section{Análisis de cadmio}

Los análisis de $\mathrm{Cd}$ en muestras de suelo agrícola se realizaron bajo el método AAA-PE-S011 (ANAVANLAB, 2019) (Anexos 4, 5), en el laboratorio Analítica Avanzada - Asesoría y Laboratorios ANAVANLAB CIA. LTDA., el mismo que está acreditado por el Servicio de Acreditación Ecuatoriano (SAE) bajo la Norma NTE INEM ISO /IEC 17025:2006.

\section{Preparación de muestras}

Previo al análisis de las muestras en el laboratorio, estas se mantuvieron a temperatura ambiente entre (15 a 30) ${ }^{\circ} \mathrm{C}$. Posteriormente se ubicó el código de
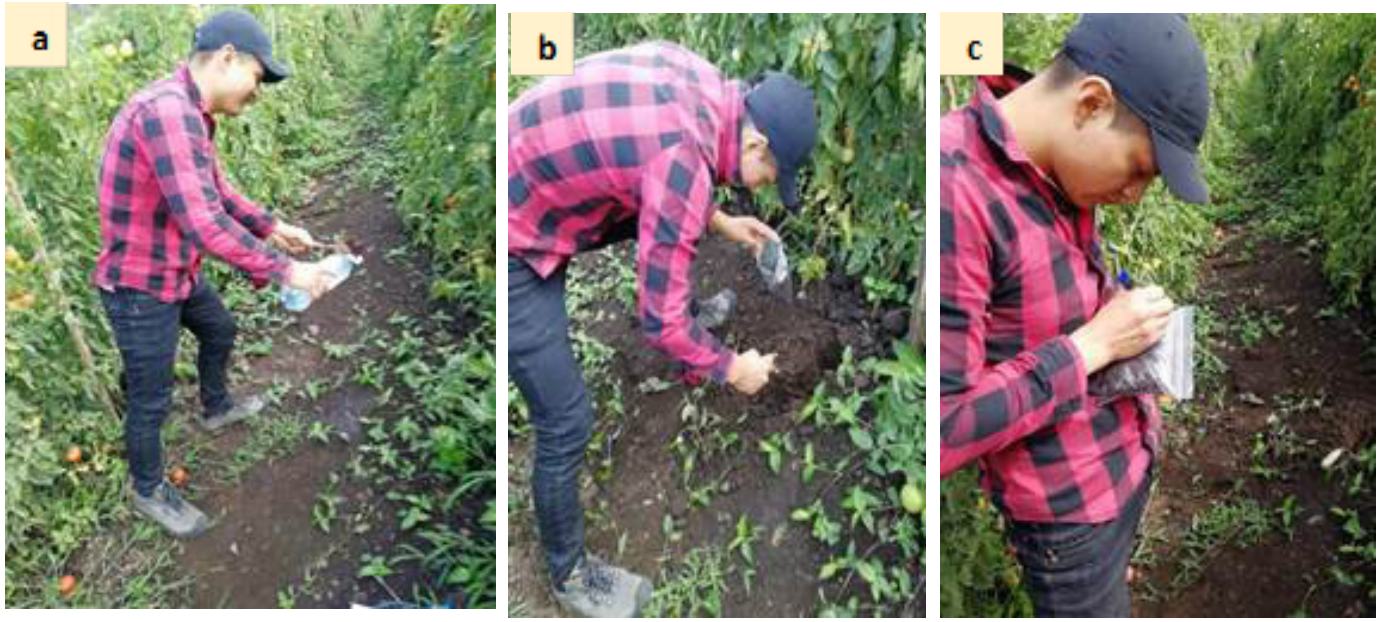

Figura 3. Metodología de campo utilizado para la extracción de muestras de suelo agrícola: a) Esterilización de utensilios b) Colecta de muestras c) Rotulado. 
cada muestra proveniente del empaque de estos a cada material volumétrico, de vidrio o auxiliar, en donde la muestra, sus diluciones o sus preparaciones fueron colocadas, de tal forma que se mantuvo reconocido en el transcurso del análisis.

Se homogeneizó cada muestra en una superficie plana retirando todo el material extraño al suelo como: rocas, hojas, insectos, etc. Continuando con el mezclado de la muestra, se procedió a dividir en cuatro porciones similares, tomando dos porciones opuestas y volviendo a realizar un cuarteo hasta llegar a una porción de aproximadamente $10 \mathrm{~g}$.

Las muestras se secaron en una estufa a una temperatura de $40{ }^{\circ} \mathrm{C} \pm 4{ }^{\circ} \mathrm{C}$ por 12 horas. Para el análisis por espectrofotometría de absorción atómica de llama en suelos, las muestras se trataron previamente con una digestión ácida asistida por microondas.

\section{Digestión ácida asistida por microondas}

Realizado bajo la referencia del método EPA 3051: Digestión Ácida asistida por Microondas de sedimentos, lodos, suelos y aceites, consistiendo básicamente en los siguientes parámetros:

Se pesó 0,50 g de muestra en un vial de digestión, y de la misma forma para duplicados y fortificaciones. Se añadió $10 \mathrm{ml}$ de ácido nítrico concentrado a cada vial y para el blanco con ácido nítrico solamente. Posteriormente, se ubicó el disco de liberación de presión sobre los viales. Una vez realizado esto se encendió el microondas y con el computador se ingresó al software "Transform 800" escogiendo en el menú principal el método EPA 3051_1.

Una vez terminado el programa, se dejó enfriar el equipo hasta $60^{\circ} \mathrm{C}$, prosiguiendo con la extracción de los viales que se terminaron de enfriar completamente al ambiente. Los viales fueron llevados a una campana de extracción, fueron abiertos con mucho cuidado y transferidos a un embudo de vidrio con papel filtro. El filtrado se recogió en un balón aforado de $25 \mathrm{ml}$ clase $A$, y finalmente se realizó un lavado con agua desionizada.

\section{Espectrofotometría de absorción atómica a llama} Basado en el método estándar de la EPA 7000 A: Métodos de Absorción Atómica, una vez que se realizó la preparación del equipo de espectrometría de absorción atómica modelo AA-EL-501 bajo el método antes mencionado se prosiguió con el encendido del computador del equipo ingresando al software "Winlab 32 for AA".

Al inicio el software se activó la función de auto chequeo para comprobar que no exista ningún tipo de error en el equipo. Una vez realizadas las pruebas iniciales, se puso al equipo en modo llama, prosiguiendo con la introducción del mechero a base de aire y acetileno.
Se prosiguió con el ajuste del quemador del equipo, para ello se encendió la lámpara de cobre y con un papel blanco se observó el haz de luz de la lámpara de Cu en el centro de la línea de la llama del mechero. Se apagó la lámpara de cobre y a la vez se encendió la lámpara para la determinación de $\mathrm{Cd}$, dejando encendida 10 minutos para su estabilización.

Posteriormente, las muestras fueron sometidas a la digestión ácida asistida por microondas. Se colocaron en el equipo siendo atomizadas y a su vez dirigidas a través de la llama pasando por el monocromático, llegando al detector que midió la cantidad de luz absorbida por el Cd atomizado y finalmente obteniendo la concentración de Cd en mg/kg.

\section{Análisis físico}

El análisis físico se realizó en el Laboratorio de Química General de la facultad de Ciencias Naturales de la Universidad de Guayaquil, tomando como referencia las metodologías estándares para cada parámetro:

\section{Color del suelo}

Se realizó a través del método de las tablas de Munsell (Castro, 2018). El cual se fundamentó en la colorimetría del sustrato. Para emplear esta técnica se tomó un puñado de suelo previamente secado al ambiente por 24 horas y se lo ubicó sobre una superficie de color blanca para tener una mayor refracción del color (Fig. 4).

\section{Textura}

Se realizó la determinación de la textura mediante el método "Feel” (Ritchey, Mcgrath, \& Gehring, 2015). Antes de realizar el procedimiento se retiró cualquier tipo de impureza que pudiera afectar los resultados, como rocas, raíces, insectos, etc. Posteriormente se situó una porción de suelo sobre la palma de una mano, se agregó una pequeña porción de agua lo suficiente para que la muestra esté húmeda, pero a su vez no muy espesa. Finalmente, se frotó la muestra húmeda entre el pulgar y los dedos sintiendo la adherencia, la arenilla, la suavidad y otras características (Fig. 5).

\section{Temperatura del suelo}

La medición de este parámetro se realizó in situ,

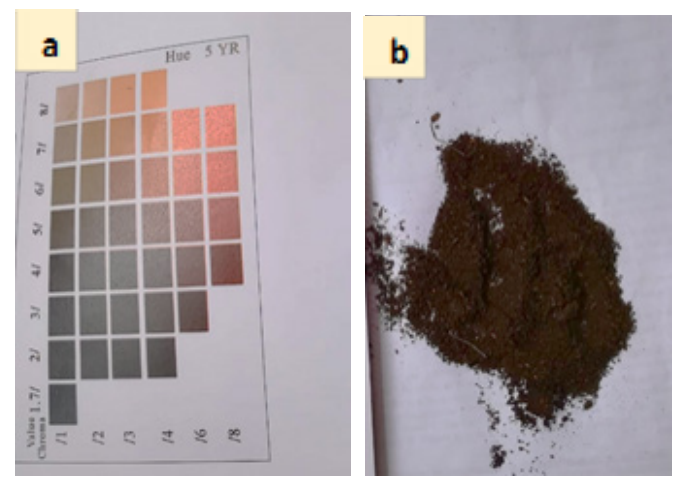

Figura 4. Determinación del color del suelo: a) tablas de Munsell b) muestra preparada para la determinación de su color. 
Rev. Cient. Cien. Nat. Ambien. 14(1):151-172 Junio 2020
Pilco \& García - Determinación de cadmio en suelos agrícolas del cantón Pallatanga, provincia de Chimborazo, Ecuador consistió en introducir el multiparamétrica de la marca "WANGCA" modelo 12541 en el suelo por aproximadamente un minuto para evitar cualquier tipo de error de medición (Fig. 7).

\section{Porcentaje de humedad}

Realizado por gravimetría a través del método AS-05 (SEMARNAT, 2002). Se colocó una porción de suelo sobre un recipiente resistente al calor previamente tarado, con la utilización de una balanza de presión de 0,01 se pesó $50 \mathrm{~g}$ de muestra humeda y con mucho cuidado se introdujo la muestra dentro del horno de secado durante 24 horas, a una temperatura de $110^{\circ}$ $\pm 5^{\circ} \mathrm{C}$. una vez transcurrido este tiempo se volvió a pesar la muestra más el peso del recipiente (Fig. 6). El porcentaje de humedad se calculó con la siguiente fórmula que fue desarrollada por la Secretaría de Medio Ambiente y Recursos Naturales de México (2002):

$$
\theta g=\frac{(P B+P s h)-(P B+P s s)}{(P B+P s s)-P B} \times 100
$$

En donde:

$\mathrm{PB}=$ Peso del bote con tapa $(\mathrm{g})$.

Psh $=$ Peso del suelo húmedo $(\mathrm{g})$.

$\mathrm{PB}+\mathrm{Psh}=$ Peso del bote más peso del suelo húmedo $(\mathrm{g})$.

PB + Pss = Peso del bote más peso del suelo seco $(\mathrm{g})$.

Los resultados del contenido de humedad fueron interpretados considerando las constantes hídricas y de humedad establecidos por Sierra (2019) (Tabla 2).
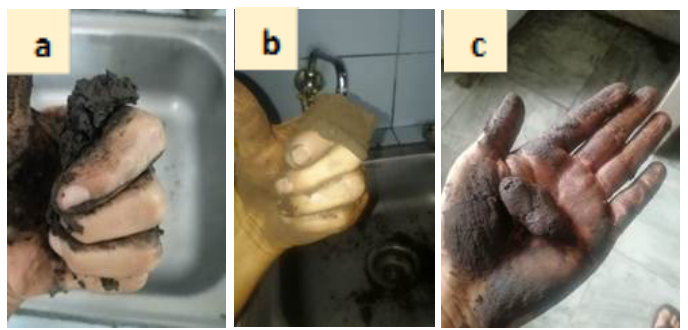

Figura 5. Determinación de la textura del suelo: a) Suelo franco arenoso b) Suelo franco limoso c) Suelo franco arcilloso limoso.

\section{Análisis químico}

$\mathrm{pH}$

Consistió en introducir el sensor del multiparamétrico "WANGCA" modelo 12541 en el sustrato por alrededor de un minuto, hasta que el resultado se quedara estable. Determinado en el mismo lugar de la toma de muestras para mayor confiabilidad de los datos (Fig. 7).

Los resultados obtenidos de $\mathrm{pH}$ del suelo fueron interpretados utilizando el criterio de la norma oficial Mexicana NOM-021-RECNAT-2000 (Tabla 3).

\section{Porcentaje de materia orgánica}

Este porcentaje fue determinado con el método gravímetro de volatilización indirecta adaptado

Tabla 2. Constantes hídricas y humedad aprovechable según la textura del suelo (Sierra, 2019).

\begin{tabular}{cccccc}
\hline Textura & $\begin{array}{c}\text { Capacidad } \\
\text { campo }\end{array}$ & $\begin{array}{c}\text { Punto } \\
\text { marchitez } \\
\text { permanente }\end{array}$ & Saturación & $\begin{array}{c}\text { Agua } \\
\text { disponible }\end{array}$ & $\begin{array}{c}\text { Rango humedad } \\
\text { aprovechable con 50\% } \\
\text { de humedad agotada }\end{array}$ \\
\hline Arcilloso & 42 & $\%$ & $\%$ & $\mathrm{Cm} / \mathrm{cm}$ & $\%$ \\
\hline Franco & 27 & 12,6 & 49 & 0,12 & $42-36$ \\
\hline Arenoso & 9 & 5 & 46 & 0,14 & $27-20$ \\
\hline
\end{tabular}
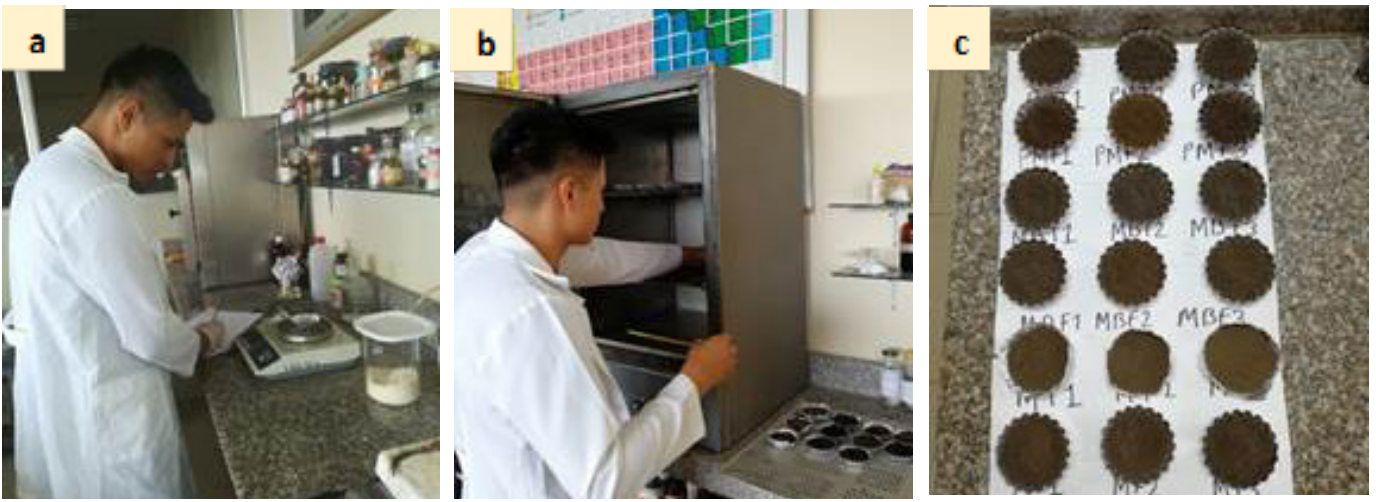

Figura 6. Determinación del porcentaje de humedad: a) pesado de muestras b) introducción de las muestras al horno de secado c) muestras post secado. 
Rev. Cient. Cien. Nat. Ambien. 14(1):151-172 Junio 2020
Pilco \& García - Determinación de cadmio en suelos agrícolas del cantón Pallatanga, provincia de Chimborazo, Ecuador por Wildlands School (2010), está basado en el método experimental de Miyazawa, Pavan, Oliveira, Ionashiro, \& Silva (2000)using several brazilian soils with variable chemical and physical properties. Gravimetric method was positively correlated with Walkley-Black method with the following linear regression equation: $y=3.72 x+0.29, r=0.94$. The angular coefficient 3.72 for tropical soils was greater than those reported in the literature for temperate soils (from 1.68 to 2.13 . El primer procedimiento realizado fue el secado de las muestras en un horno durante 24 horas, a una temperatura de $110^{\circ} \pm 5^{\circ} \mathrm{C}$.

Una vez realizado esto se prosiguió a pesar $10 \mathrm{~g}$ de suelo en recipientes resistentes al calor previamente tarados y rotulados. Posteriormente, se prosiguió a someter las muestras a fuego directo por 30 minutos. Para evitar que la humedad del ambiente interfiera en el procedimiento de calcinación los recipientes permanecieron tapados e inmediatamente terminado el proceso, fueron pesadas (Fig. 8). Para el cálculo del porcentaje de materia orgánica se utilizó la siguiente formula que fue desarrollada por la Secretaría de Medio Ambiente y Recursos Naturales de México (2002):

En donde:

$$
\% \mathrm{MO}=\frac{\mathrm{P} 1-\mathrm{P} 2}{\mathrm{P} 2-\mathrm{C}} \times 100
$$

P1 = Peso de la muestra antes de la calcinación. P2 = Peso de la muestra después de la calcinación. $\mathrm{C}=$ Peso del recipiente.

Los resultados de porcentaje de materia orgánica del suelo fueron interpretados utilizando el criterio de la norma oficial Mexicana NOM-021-RECNAT-2000 (Tabla 4).

\section{Conductividad eléctrica}

Para el análisis de este parámetro se utilizó el metodología diseñada por Sonmez, Buyuktas, Okturen Asri, \& Citak (2008). Se prosiguió a pesar $50 \mathrm{~g}$ de suelo y $50 \mathrm{ml}$ de agua destilada que posteriormente se homogenizó con la utilización de una barrilla de vidrio. Una vez realizado la disolución de extracto suelo/agua 1:1 se prosiguió a sumergir el sensor del multiparamétrico de la marca "APERA INTRUMENTS" modelo PC60 para realizar las respectivas mediciones. Los resultados de la conductividad eléctrica fueron interpretados utilizando el criterio de la norma oficial Mexicana NOM-021-RECNAT-2000 (Tabla 5).

\section{Tratamiento de los datos}

Los datos fueron ordenados y tabulados en una hoja de cálculo de Excel, del sistema operativo Microsoft Windows Office 365 del 2019, para posteriormente realizar el procesamiento numérico con ayuda del software Statgraphics Centurión XVI.I.

Se realizó la comprobación de los supuestos de ANOVA a cada una de las variables a estudiar y se
Tabla 3. Clasificación del suelo en cuanto a su valor de pH (SEMARNAT, 2002).

\begin{tabular}{cc}
\hline Clasificación & $\mathrm{pH}$ \\
\hline fuertemente ácido & $<5,0$ \\
moderadamente ácido & $5,1-6,5$ \\
neutro & $6,6-7,3$ \\
medianamente alcalino & $7,4-8,5$ \\
fuertemente alcalino & $>8,5$ \\
\hline
\end{tabular}
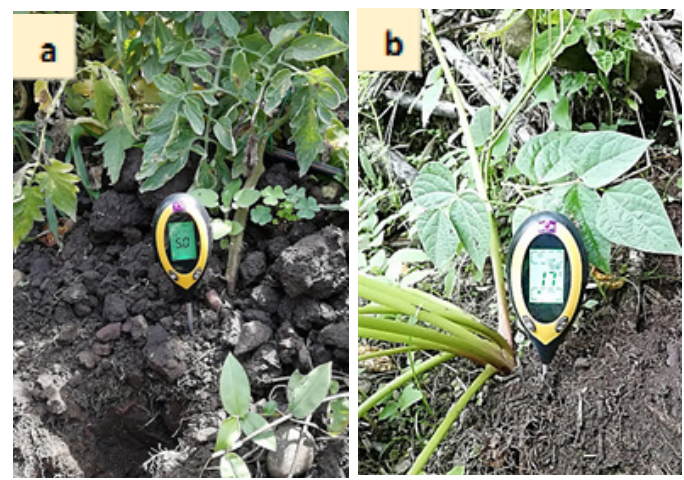

Figura 7. Medición de los parámetros fisicoquímicos del suelo con el uso del multiparamétrico "WANGCA" modelo 12541: a) medición del $\mathrm{pH}$ b) medición de la temperatura.

Tabla 4. Criterio de interpretación del porcentaje de materia orgánica del suelo (SEMARNAT, 2002).

\begin{tabular}{ccc}
\hline Clase & \multicolumn{2}{c}{ Materia orgánica $(\%)$} \\
\hline & $\begin{array}{c}\text { suelos } \\
\text { volcánicos }\end{array}$ & $\begin{array}{c}\text { suelos no } \\
\text { volcánicos }\end{array}$ \\
\cline { 2 - 3 } Muy bajo & $<4,0$ & $<0,5$ \\
Bajo & $4,1-6,0$ & $0,6-1,5$ \\
Medio & $6,1-10,9$ & $1,6-3,5$ \\
Alto & $11,0-16,0$ & $3,6-6,0$ \\
Muy alto & $>16,1$ & $>6,0$ \\
\hline
\end{tabular}
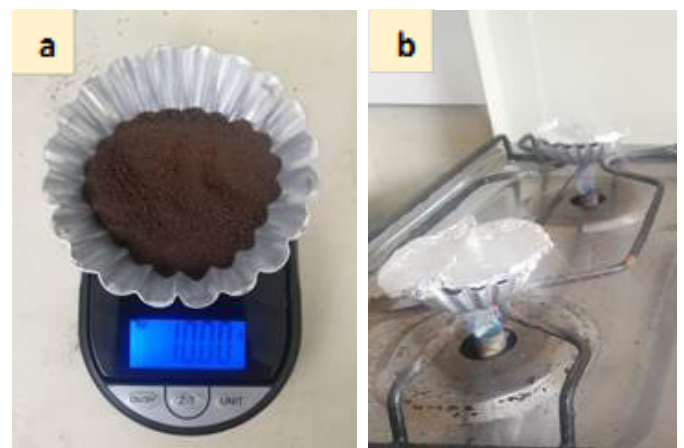

Fig. 8. Determinación de materia orgánica del suelo: a) pesado de la muestra b) calcinación de la muestra 
Rev. Cient. Cien. Nat. Ambien. 14(1):151-172 Junio 2020
Pilco \& García - Determinación de cadmio en suelos agrícolas del cantón Pallatanga, provincia de Chimborazo, Ecuador
Tabla 5. Criterio de interpretación de la conductividad eléctrica del suelo (SEMARNAT, 2002).

\begin{tabular}{cc}
\hline CE dS/m & Efectos \\
\hline 1,0 & $\begin{array}{c}\text { Efectos } \\
\text { despreciables de la } \\
\text { salinidad } \\
\text { Muy ligeramente } \\
\text { salino } \\
1,1-2,0\end{array}$ \\
$2,1-4,0$ & $\begin{array}{c}\text { Moderadamente } \\
\text { salino } \\
4,1-8,0\end{array}$ \\
$8,1-16,0$ & $\begin{array}{c}\text { Suelo salino } \\
\text { Fuertemente salino } \\
>16,0\end{array}$ \\
\end{tabular}

comprobó que no poseen una distribución normal con la prueba estadística de Anderson Darling según Zar (1996; Anexo 3). Para comparar los resultados obtenidos de Cd y del análisis fisicoquímico, para dos variables independientes (meses y cultivos) se aplicó la prueba no paramétrica de Mann Whitney (W) según Sokal \& Rohlf (2002), y para tres o más variables independientes (pisos altitudinales) se utilizó la prueba no paramétrica de Kruskal Wallis $(\mathrm{KW})$, tomando el valor de $\mathrm{p}<0,05$ para determinar si existen diferencias estadísticamente significativas, las mismas que fueron representadas por una ilustración de caja de bigote según las recomendaciones (Boyer, Fourqurean, \& Jones, 1997).

Se realizó un Análisis de Componentes Principales (ACP) para evaluar qué variable ambiental incide en la concentración de Cd (Cuadras, 1983), se tomó como referencia las correlaciones entre un conjunto de datos, y finalmente se comparó los rangos absolutos de Spearman de una proyección ortogonal (Evans, 1996; Tabla 6).

\section{Resultados}

Concentración de cadmio del mes de noviembre y diciembre

Los valores promedios de cadmio durante noviembre y diciembre de 2019 no muestran una variación marcada con respecto a la concentración del metal,

Tabla 6. Rangos absolutos de la correlación de Spearman (Evans, 1986).

\begin{tabular}{cc|c}
\hline \multicolumn{2}{c}{ Rangos } & Interpretación \\
\hline 0 & 0,19 & Muy débil \\
0,20 & 0,39 & Débil \\
0,40 & 0,59 & Moderado \\
0,60 & 0,79 & Fuerte \\
0,80 & 1 & Muy fuerte \\
\hline
\end{tabular}
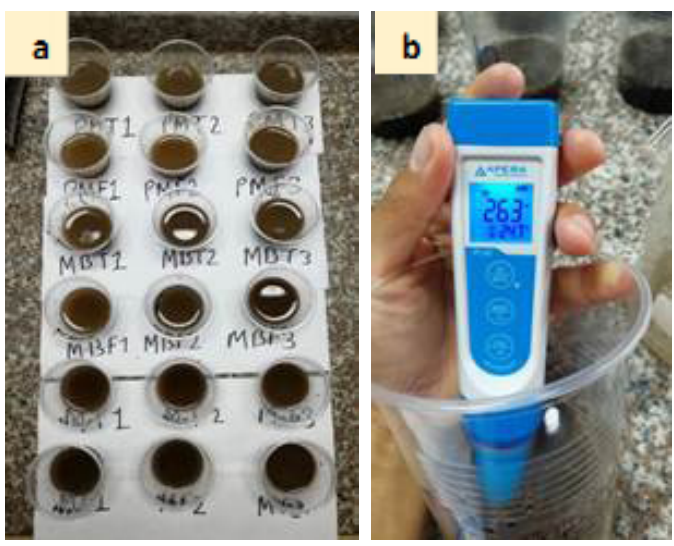

Fig. 9. Determinación de la conductividad eléctrica: a) disoluciones de extracto de suelo/agua 1:1 b) medición de la conductividad eléctrica.

registrándose en diciembre un valor de $0,80 \pm 0,11$ $\mathrm{mg} / \mathrm{kg}$; seguido de noviembre con $0,77 \pm 0,13 \mathrm{mg} / \mathrm{kg}$. De acuerdo con el criterio de calidad del suelo de uso agrícola del TULSMA Libro VI anexo 2, estos valores promedios sobrepasan el límite máximo permisible de $0,5 \mathrm{mg} / \mathrm{kg}$, pero se encuentran bajo los lineamentos según la normativa internacional de Holanda de $0,8 \mathrm{mg} / \mathrm{kg}$, que están representadas con una línea entrecortada roja (Fig. 10); mientras el valor máximo de concentración de $\mathrm{Cd}$ en el suelo se registró en diciembre 2019 con $1 \mathrm{mg} / \mathrm{kg}$.

Estos valores no representan diferencias estadísticamente significativas ( $W=178,5 ; p=0,599429)$ entre las concentraciones de $\mathrm{Cd}$ durante noviembre y diciembre de 2019 (Fig. 11).

Concentración de cadmio de los pisos altitudinales Los valores promedio del Cd con los pisos altitudinal piemontano, montano bajo y montano se encontraron entre rangos de $0,71 \pm 0,12 \mathrm{mg} / \mathrm{kg}, 0,85 \pm 0,08 \mathrm{mg} / \mathrm{kg}$ y $0,80 \pm 0,12 \mathrm{mg} / \mathrm{kg}$ respectivamente. Sobrepasando el límite máximo permitido a nivel nacional de $0,5 \mathrm{mg} /$ kg para calidad de suelo de uso agrícola, pero con la normativa internacional (LMP de $0,8 \mathrm{mg} / \mathrm{kg}$ ), únicamente el piso altitudinal montano bajo se encuentra fuera de los límites, siendo estos representados con una línea entrecortada roja (Fig. 12).

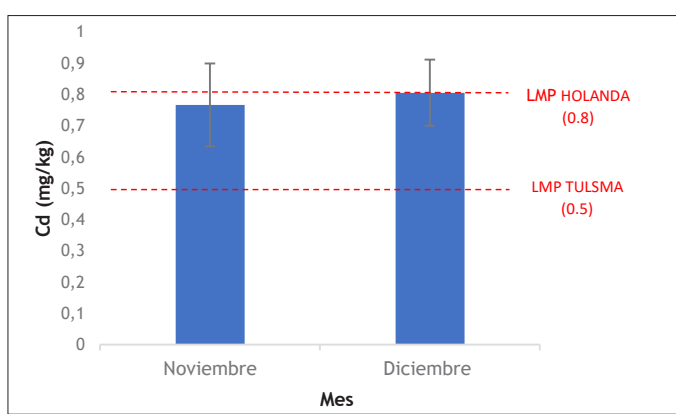

Figura 10. Concentración del cadmio registrada durante los meses noviembre y diciembre de 2019 en el cantón Pallatanga. Las barras representan el promedio \pm error estándar. 
Rev. Cient. Cien. Nat. Ambien. 14(1):151-172 Junio 2020
Pilco \& García - Determinación de cadmio en suelos agrícolas del cantón Pallatanga, provincia de Chimborazo, Ecuador

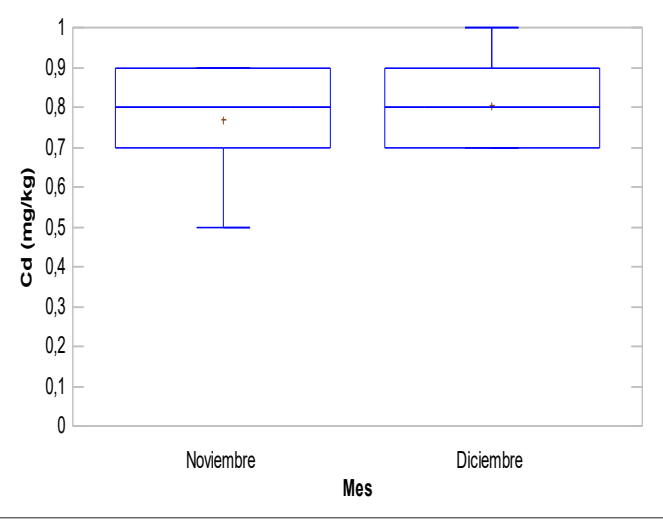

Figura 11. Diferencias significativas de la concentración de Cd durante los meses noviembre y diciembre de 2019, tomando como referencia $\mathrm{p}<0,05$ según Mann Whitney.

El mayor valor promedio de Cd en suelos agrícolas se obtuvo en el piso altitudinal montano bajo, sin embargo, el piso montano registró la máxima concentración individual de $1 \mathrm{mg} / \mathrm{kg} \mathrm{Cd}$, este resultado reflejó diferencias estadísticamente significativas ( $\mathrm{KW}=8,26$; $\mathrm{p}=0,02$ ) entre la concentración de cadmio en relación con los pisos altitudinales estudiados (Fig. 13).

\section{Concentración de cadmio de los cultivos}

La concentración promedio de Cd para el cultivo de tomate (Solanum lycopersicum) se estableció en $0,81 \pm 0,08 \mathrm{mg} / \mathrm{kg}$; mientras que en el cultivo del fréjol (Phaseolus vulgaris) fue $0,76 \pm 0,15 \mathrm{mg} / \mathrm{kg}$. De igual manera, estos resultados sobrepasan el límite de tolerancia del reglamento nacional, pero con respecto a la norma internacional se encuentra dentro del límite establecido, los cuales están representados con una línea roja entrecortada (Fig. 14).

Cabe indicar que el fréjol registró una máxima concentración de $1 \mathrm{mg} / \mathrm{kg}$, y el tomate registró una máxima de $0,9 \mathrm{mg} / \mathrm{kg}$, en donde no se presentó

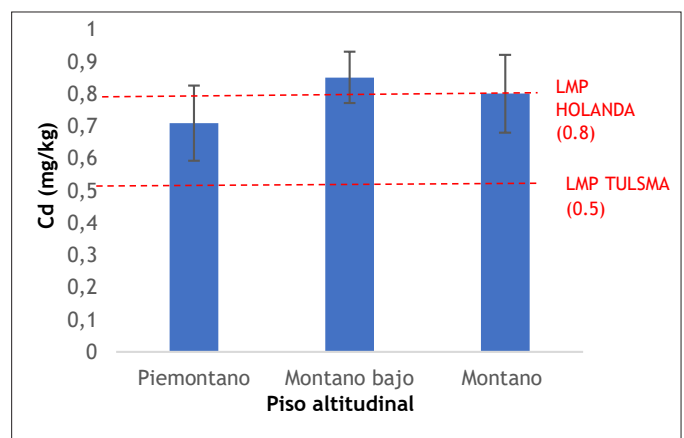

Figura 12. Concentración del cadmio de los pisos altitudinales estudiados en el cantón Pallatanga. Las barras representan el promedio \pm error estándar. diferencias estadísticamente significativas ( $W=123,0$; $p=0,204598$ ) entre la concentración de cadmio con los cultivos (Fig. 15).

\subsubsection{Valor del $\mathrm{pH}$ del mes de noviembre $\mathrm{y}$ diciembre}

El valor promedio del $\mathrm{pH}$ obtenido de los suelos agrícolas de los cultivos estudiados muestran que los suelos son moderadamente ácidos. En noviembre de 2019 se registró un promedio de $6,08 \pm 0,90$; mientras

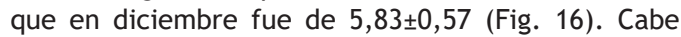
indicar que estos valores no presentan diferencias estadísticamente significativas $(W=121,0 ; p=0,190225)$ del $\mathrm{pH}$ durante los meses de estudio (Fig. 17).

\section{Valor del $\mathrm{pH}$ de los pisos altitudinales}

El valor promedio del $\mathrm{pH}$ obtenido entre los pisos altitudinales tiene su máxima representación en el piso montano con $6,5 \pm 0,56$, mientras que el piso piemontano obtuvo el menor valor con $5,29 \pm 0,66$; estos rangos son considerados moderadamente ácidos. Los resultados obtenidos indican que existen diferencias estadísticamente significativas ( $K W=16,21 ; p=0,0003$ ) entre los pisos altitudinales muestreados.

\section{Valor del pH de los cultivos}

El valor promedio de $\mathrm{pH}$ fue de $5,94 \pm 0,84$ para el suelo del cultivo de tomate, y 5,97 $\pm 0,67$ para el cultivo de fréjol, siendo estos catalogados como suelos moderadamente ácidos (Fig. 20). Con la realización de la prueba no paramétrica de Mann Whitney se comprobó que no existe una diferencia estadísticamente significativa ( $W=160,5 ; p=0,974192)$ entre el $\mathrm{pH}$ con relación a los cultivos estudiados (Fig. 21).

\section{Porcentaje de materia orgánica del mes de} noviembre y diciembre

Para el mes de noviembre el porcentaje de materia orgánica mostró un promedio de $6,96 \pm 1,66$, y para diciembre $6,66 \pm 1,68$. Los porcentajes encontrados se catalogaron como clase media en materia orgánica, es decir, estuvieron dentro del rango de 6,1 y 10,9 para los suelos de tipo volcánico durante los dos meses de estudio (Fig. 22). No existe una diferencia estadísticamente significativa $(W=133,0 ; p=0,367184)$

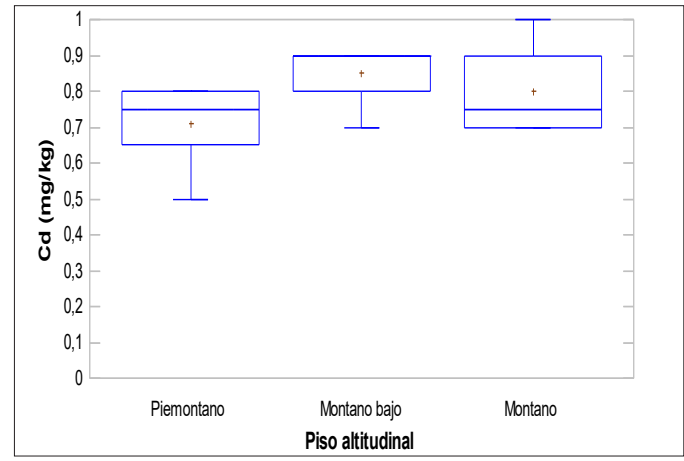

Figura 13. Diferencias significativas de los pisos altitudinales con la concentración de Cd, tomando como referencia $p<0,05$ según Kruskal Wallis 
Rev. Cient. Cien. Nat. Ambien. 14(1):151-172 Junio 2020
Pilco \& García - Determinación de cadmio en suelos agrícolas del cantón Pallatanga, provincia de Chimborazo, Ecuador

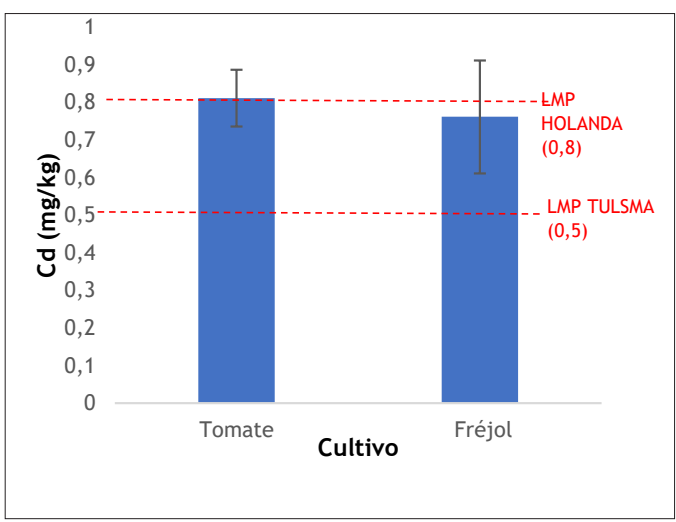

Figura 14. Concentración de cadmio de los cultivos de tomate y fréjol estudiados en el cantón Pallatanga. Las barras representan el promedio \pm error estándar.

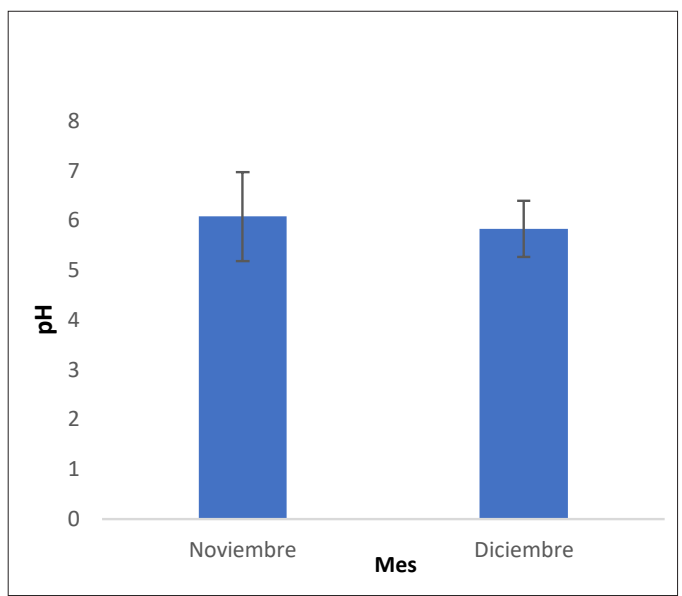

Figura 16. Valor del pH durante noviembre y diciembre de 2019 en el cantón Pallatanga. Las barras representan el promedio \pm error estándar.

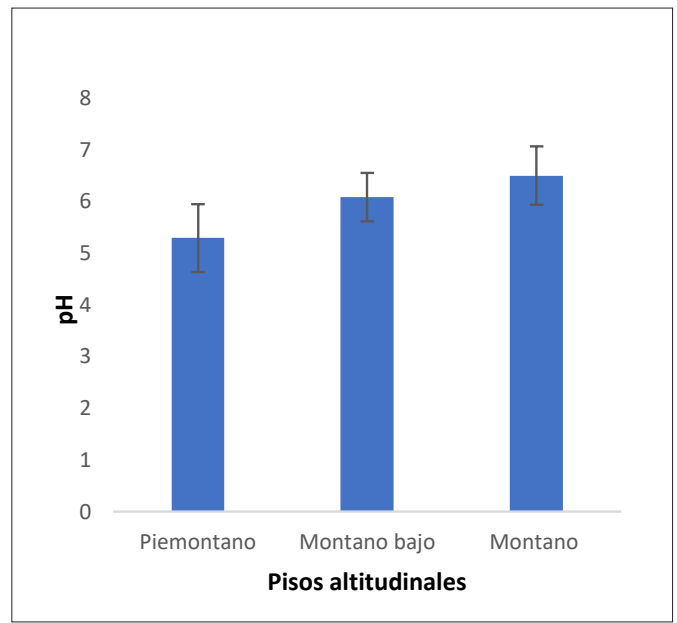

Figura 18. Valor del pH de los pisos altitudinales en el cantón Pallatanga. Las barras representan el promedio \pm error estándar.

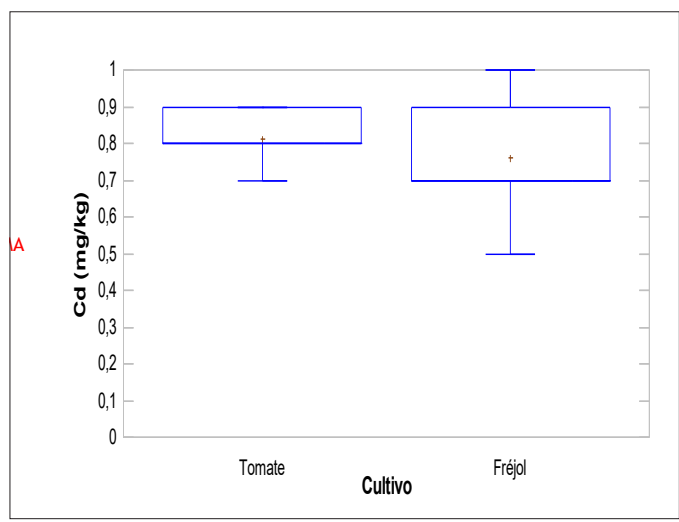

Figura 15. Diferencias significativas de la concentración de $\mathrm{Cd}$ en cultivos de tomate $\mathrm{y}$ fréjol, tomando como referencia $\mathrm{p}<0,05$ según Mann Whitney.

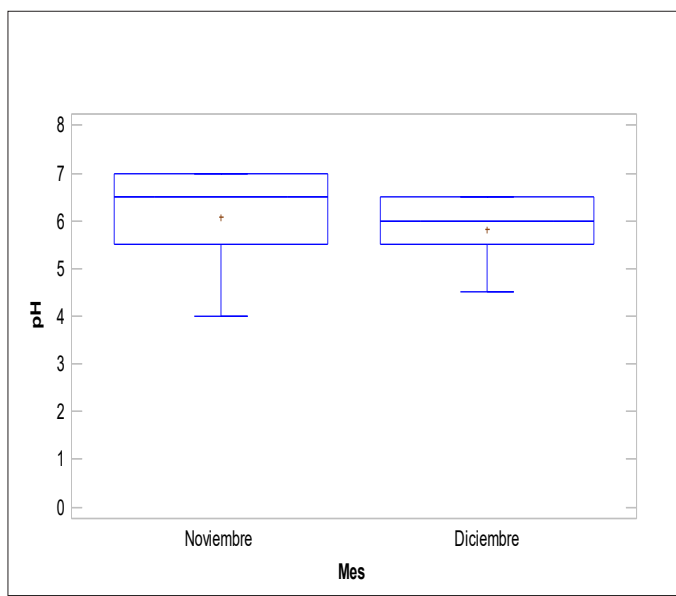

Figura 17. Diferencias significativas del valor del pH durante noviembre y diciembre de 2019, tomando como referencia $\mathrm{p}<0,05$ según Mann Whitney.

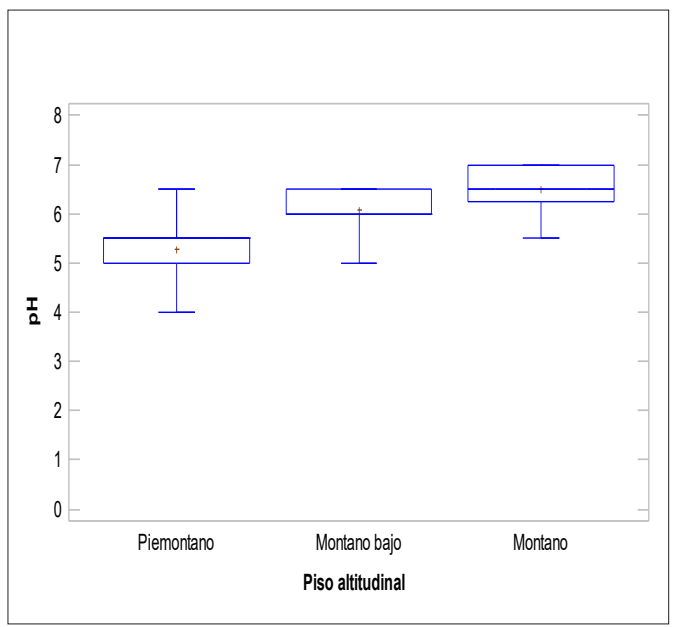

Figura 19. Diferencias significativas del $\mathrm{pH}$ según los pisos altitudinales, tomando como referencia $\mathrm{p}<0,05$ según Kruskal Wallis. 
Rev. Cient. Cien. Nat. Ambien. 14(1):151-172 Junio 2020
Pilco \& García - Determinación de cadmio en suelos agrícolas del cantón Pallatanga, provincia de Chimborazo, Ecuador

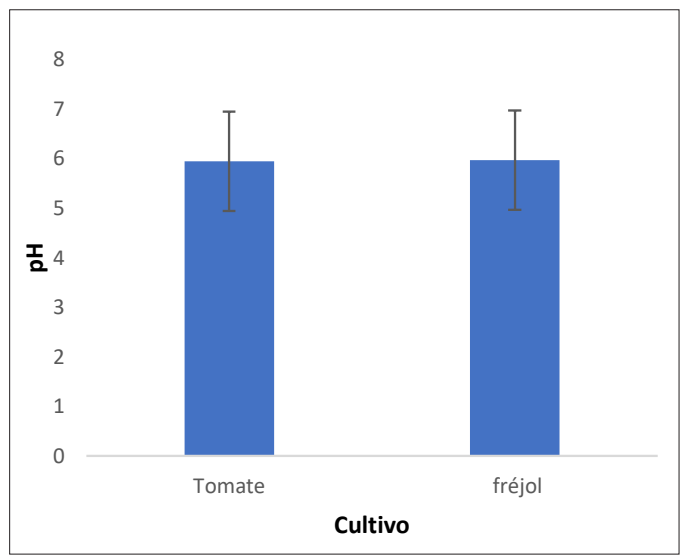

Figura 20. Valor del pH con relación al suelo de los cultivos de tomate y fréjol en el cantón Pallatanga. Las barras representan el promedio \pm error estándar.

entre las medianas del porcentaje de materia orgánica con relación a los meses estudiados (Fig. 23).

\section{Porcentaje de materia orgánica de los pisos altitudinales}

Para los pisos altitudinales el porcentaje promedio fue $5,88 \pm 0,42$ en piemontano; $7,93 \pm 2,45$ en montano bajo; y 6,60 $\pm 0,40$ en montano. Con estos porcentajes el piso piemontano se cataloga como de clase baja en materia orgánica y los pisos altitudinales restantes se encuentran en clasificación media (Fig. 24). Con la realización de la prueba de Kruskal Wallis se constató que existen diferencias estadísticamente significativas $(K W=10,82 ; p=0,004)$ para el porcentaje de materia orgánica con relación a los pisos altitudinales (Fig. 25).

\section{Porcentaje de materia orgánica de los cultivos}

El porcentaje promedio de la materia orgánica del suelo para el cultivo de tomate fue de $6,19 \pm 0,45$ mientras para el cultivo de fréjol fue de $7,42 \pm 2,15$. Siendo estos cultivos de clase media según el contenido de materia orgánica para suelos de origen volcánicos (Fig. 26). No existen diferencias estadísticamente significativas $(W=214,0 ; p=0,103208$ ) para el porcentaje de materia orgánica con relación al suelo de los cultivos (Fig. 27). Conductividad eléctrica durante los meses noviembre y diciembre

La conductiva eléctrica durante noviembre registró un promedio de $0,43 \pm 0,22 \mathrm{dS} / \mathrm{m}$, en diciembre fue de $0,56 \pm 0,48 \mathrm{dS} / \mathrm{m}$, lo cual corresponde a suelos con efectos despreciables de salinidad para ambos casos (Fig. 28). Una vez realizado la prueba de Mann Whitney, se comprobó que no existe una diferencia estadísticamente significativa $(W=162,0 ; p=0,987359)$ para la conductividad eléctrica con los meses estudiados (Fig. 29).

\section{Conductividad eléctrica de los pisos altitudinales}

Para los pisos altitudinales los valores promedios de la conductividad eléctrica fueron de 0,84 $\pm 0,49$ $\mathrm{dS} / \mathrm{m}$ para piemontano; $0,30 \pm 0,09$ para montano

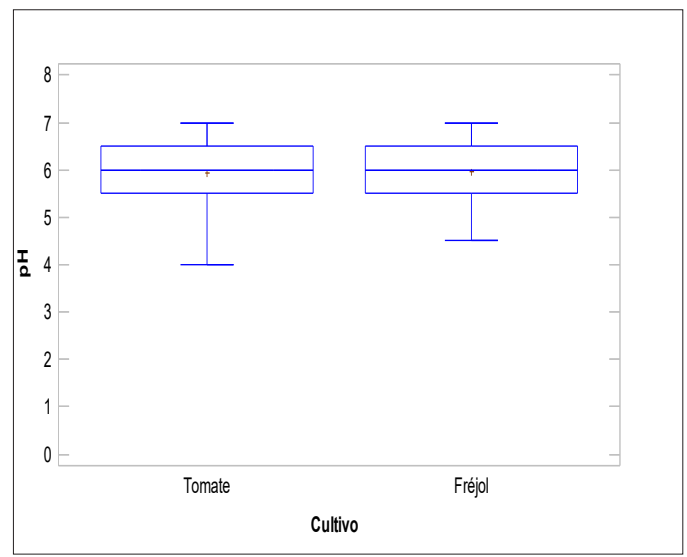

Figura 21. Diferencias significativas del valor del pH en suelos de cultivos de tomate y fréjol, tomando como referencia $p<0,05$ según Mann Whitney.

bajo; y 0,33 $\pm 0,08$ para montano, estos resultados corresponden a suelos con efectos despreciables de salinidad (Fig. 30). Sí existe una diferencia estadísticamente significativa $(\mathrm{KW}=13,73 ; \mathrm{p}=0,001)$ según la prueba de Kruskal Wallis para la conductiva eléctrica con los pisos altitudinales (Fig. 31).

\section{Conductividad eléctrica de los cultivos}

La conductividad eléctrica de los cultivos estudiados mostró un efecto despreciable de salinidad puesto que el valor promedio del suelo del tomate fue de $0,59 \pm 0,48 \mathrm{dS} / \mathrm{m}$ y para el fréjol $0,40 \pm 0,21 \mathrm{dS} / \mathrm{m}$ (Fig. 32). No existe una diferencia estadísticamente significativa ( $W=131,5 ; p=0,34201$ ) de la conductividad eléctrica con los cultivos estudiados (Fig. 33).

Temperatura del suelo durante los meses noviembre y diciembre

La temperatura del suelo para el mes de noviembre mostró un promedio de $21,16 \pm 2,54^{\circ} \mathrm{C}$ y para diciembre $20,6 \pm 2,32^{\circ} \mathrm{C}$ (Fig. 34). Con la realización de la prueba de Mann Whitney se evidenció que los resultados no presentan diferencias estadísticamente significativas $(W=162,0 ; p=0,987232)$ en relación con los meses de control (Fig. 35).

Temperatura del suelo de los pisos altitudinales La temperatura promedio del suelo para el piso piemontano estuvo en $23,67 \pm 1,44^{\circ} \mathrm{C}$; para montano bajo fue de $19,08 \pm 1,78^{\circ} \mathrm{C}$ y en el piso montano $19,92 \pm 0,66^{\circ} \mathrm{C}$, estos resultados son característicos de los suelos subtropicales en Ecuador (Fig. 36). Se realizó la prueba de Kruskal Wallis en donde se demostró que no existen diferencias estadísticamente significativas $(\mathrm{KW}=24,24 ; \mathrm{p}=0,000005)$ para la temperatura del suelo con los pisos altitudinales (Fig. 37).

\section{Temperatura del suelo de los cultivos}

La temperatura promedio del suelo en cultivo de tomate mostró un valor de $21,50 \pm 2,31^{\circ} \mathrm{C}$ y para el fréjol $20,28 \pm 2,44^{\circ} \mathrm{C}$ (Fig. 38), la prueba de Mann Whitney 
Rev. Cient. Cien. Nat. Ambien. 14(1):151-172 Junio 2020
Pilco \& García - Determinación de cadmio en suelos agrícolas del cantón Pallatanga, provincia de Chimborazo, Ecuador

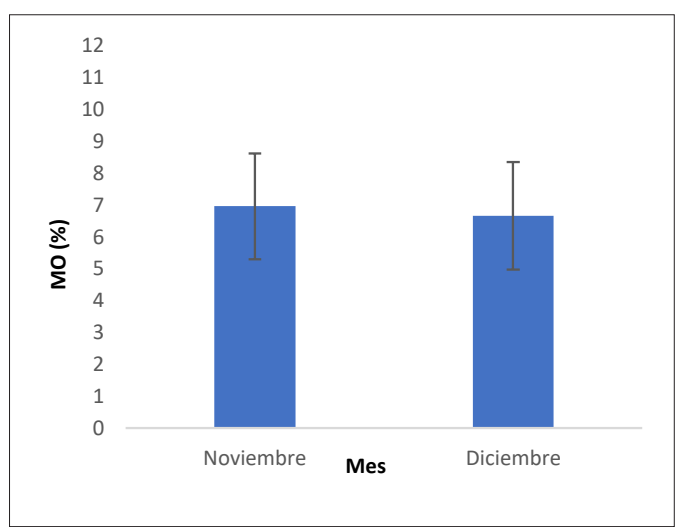

Figura 22. Porcentaje de materia orgánica durante noviembre y diciembre de 2019 en el cantón Pallatanga. Las barras representan el promedio \pm error. estándar.

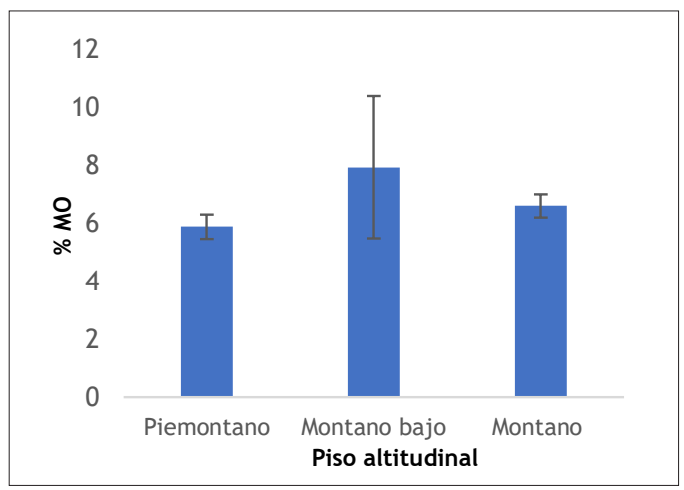

Figura 24. Porcentaje de materia orgánica por pisos altitudinales en el cantón Pallatanga. Las barras representan el promedio \pm error estándar.

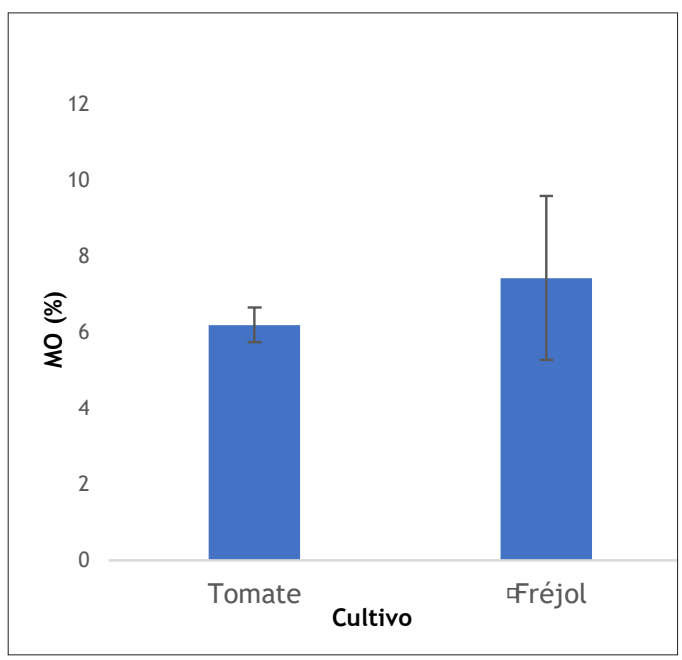

Figura 26. Porcentaje de materia orgánica en suelos de cultivo de tomate y fréjol en el cantón Pallatanga. Las barras representan las medianas \pm error estándar.

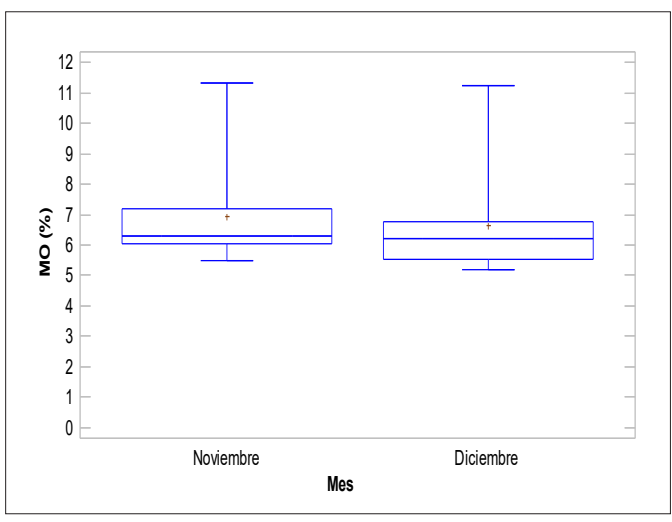

Figura 23. Diferencias significativas del porcentaje de materia orgánica durante noviembre y diciembre de 2019 , tomando como referencia $\mathrm{p}<0,05$ según Mann Whitney.

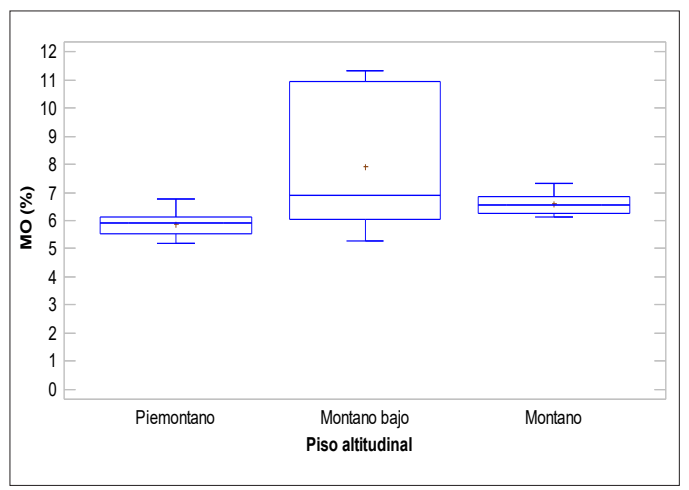

Figura 25. Diferencias significativas entre la materia orgánica y los pisos altitudinales tomando como referencia $p<0,05$ según Kruskal Wallis.

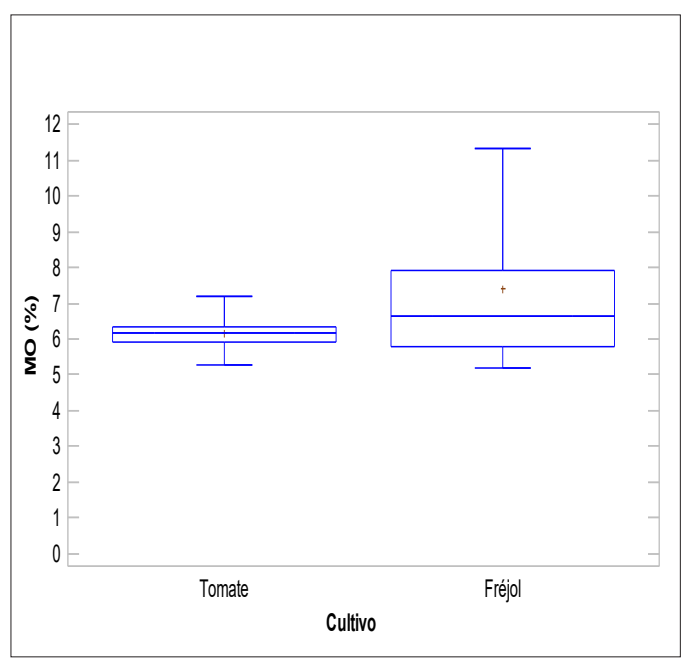

Figura 27. Diferencias significativas del porcentaje de materia orgánica en los suelos de los cultivos de tomate y fréjol, tomando como referencia $\mathrm{p}<0,05$ según Mann Whitney. 
Rev. Cient. Cien. Nat. Ambien. 14(1):151-172 Junio 2020
Pilco \& García - Determinación de cadmio en suelos agrícolas del cantón Pallatanga, provincia de Chimborazo, Ecuador

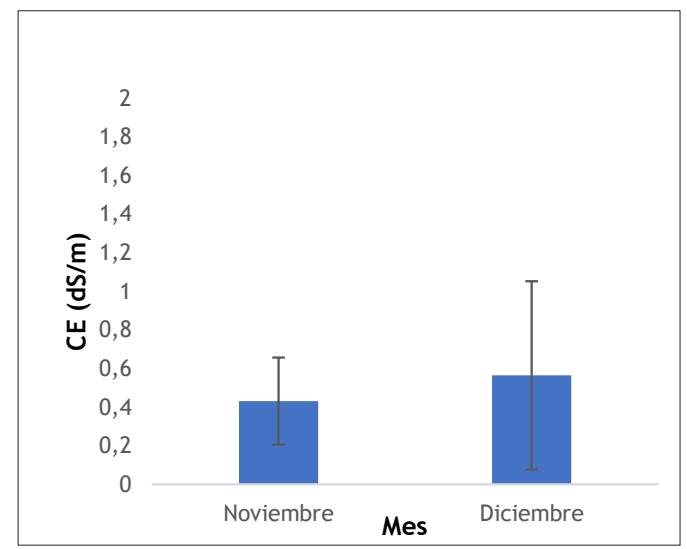

Figura 28. Conductividad eléctrica durante noviembre y diciembre de 2019 en el cantón Pallatanga. Las barras representan el promedio \pm error estándar.

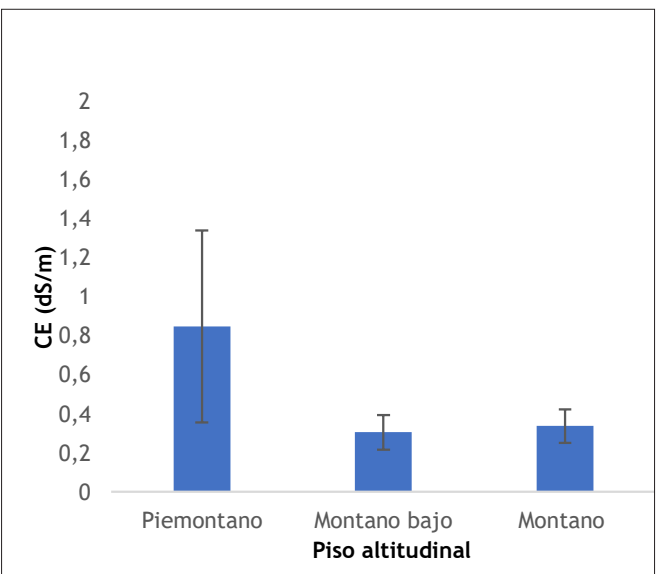

Figura 30. Conductividad eléctrica de los pisos altitudinales en el cantón Pallatanga. Las barras representan el promedio \pm error estándar.

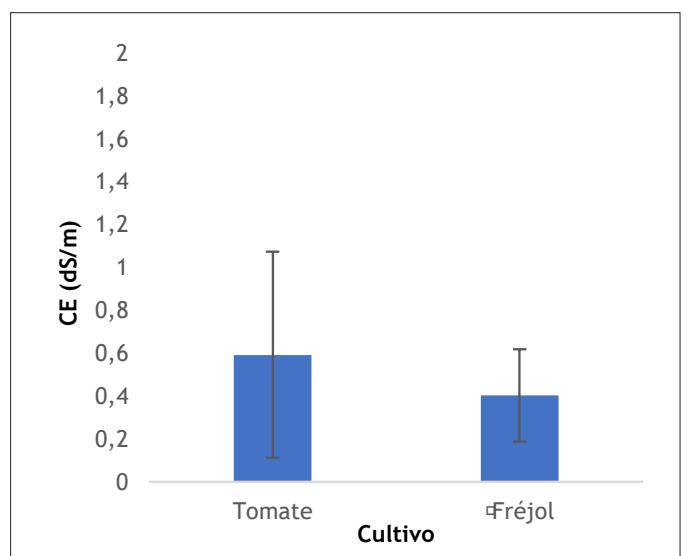

Figura 32. Conductividad eléctrica del suelo de los cultivos de tomate y fréjol en el cantón Pallatanga. Las barras representan el promedio \pm error estándar.

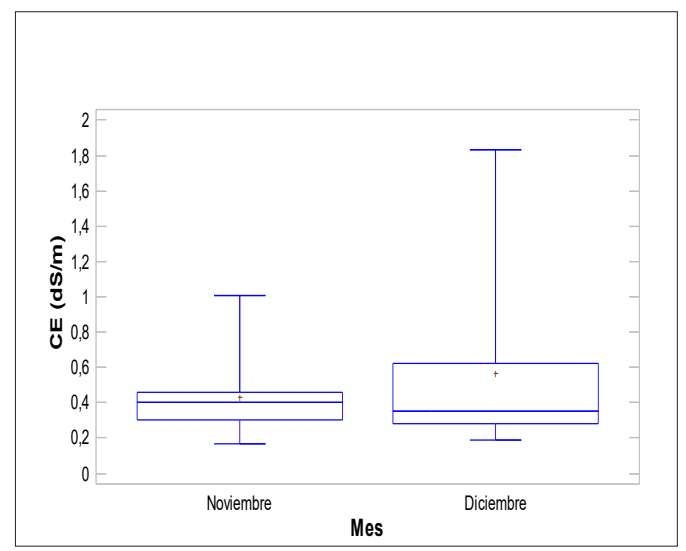

Figura 29. Diferencias en la conductividad eléctrica durante noviembre y diciembre de 2019 en el cantón Pallatanga, tomando como referencia $\mathrm{p}<0,05$ según Mann Whitney.

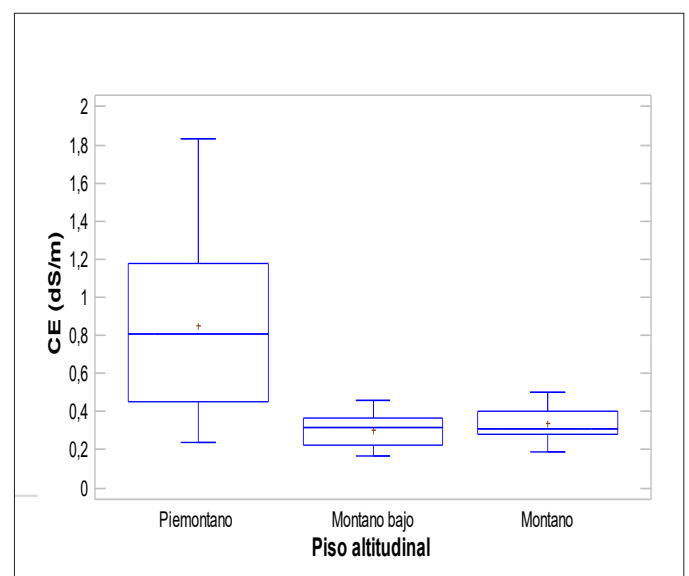

Figura 31. Diferencias significativas de los pisos altitudinales con la conductividad eléctrica tomando como referencia $\mathrm{p}<0,05$ según Kruskal Wallis.

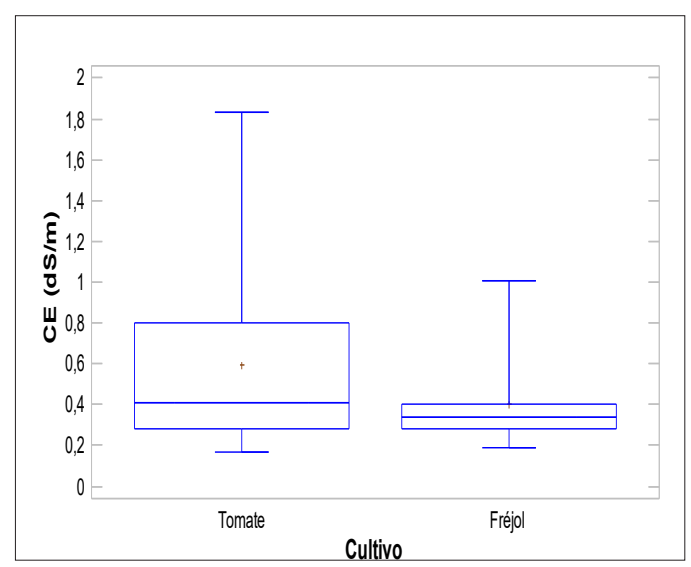

Figura 33. Diferencias significativas de los cultivos de tomate y fréjol en relación a la conductividad eléctrica, tomando como referencia $\mathrm{p}<0,05$ según Mann Whitney. 
Rev. Cient. Cien. Nat. Ambien. 14(1):151-172 Junio 2020
Pilco \& García - Determinación de cadmio en suelos agrícolas del cantón Pallatanga, provincia de Chimborazo, Ecuador

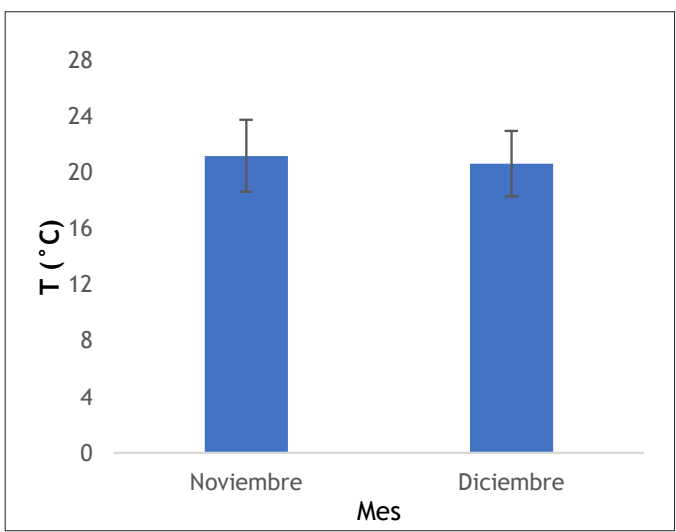

Figura 34. Temperatura del suelo en los pisos altitudinales en el cantón Pallatanga durante noviembre y diciembre de 2019. Las barras representan el promedio \pm error estándar.

no presentó diferencias estadísticamente significativas $(W=124,5 ; p=0,231795)$ para la temperatura del suelo con los cultivos (Fig. 39).

\section{Humedad del suelo durante noviembre y diciembre de 2019}

La humedad promedio del suelo durante noviembre fue $18,78 \pm 3,33 \%$ y para diciembre $21,52 \pm 7,01 \%$ (Fig. 40). Con la prueba de Mann Whitney no presentó una diferencia estadísticamente significativa ( $\mathrm{W}=\mathrm{p} 192,0$; $\mathrm{p}=0,350613$ ) para la humedad del suelo con los meses estudiados (Fig. 41).

\section{Humedad del suelo de los pisos altitudinales}

La humedad promedio para el piso altitudinal piemontano estuvo en $19,42 \pm 7,55 \%$; para montano bajo $21,30 \pm 3,30 \%$; y para montano en $19,73 \pm 5,40 \%$. Según la tabla de contantes hídricas (Sierra, 2019), el piso altitudinal montano se encuentra dentro del rango de humedad aprovechaba para los suelos de tipo franco (Fig. 42). Con la realización de la prueba Kruskal Wallis se pudo conocer que no existen diferencias estadísticamente significativas $(\mathrm{KW}=5,77 ; \mathrm{p}=0,05)$ para la humedad del suelo en comparación a los pisos altitudinales (Fig. 43).

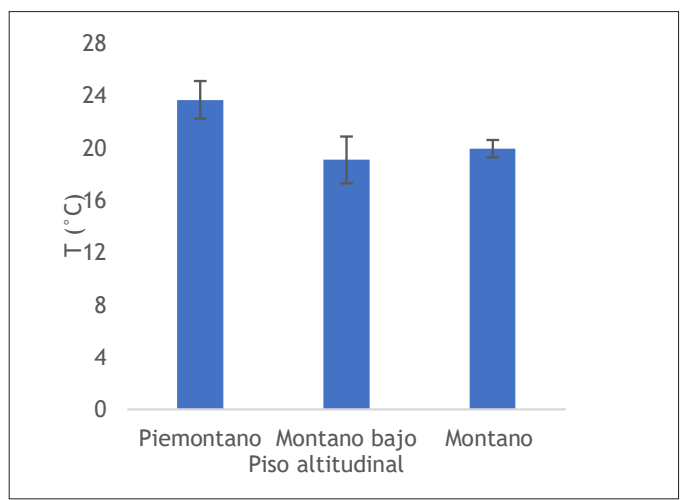

Figura 36. Temperatura del suelo por pisos altitudinales en el cantón Pallatanga. Las barras representan el promedio \pm error estándar.

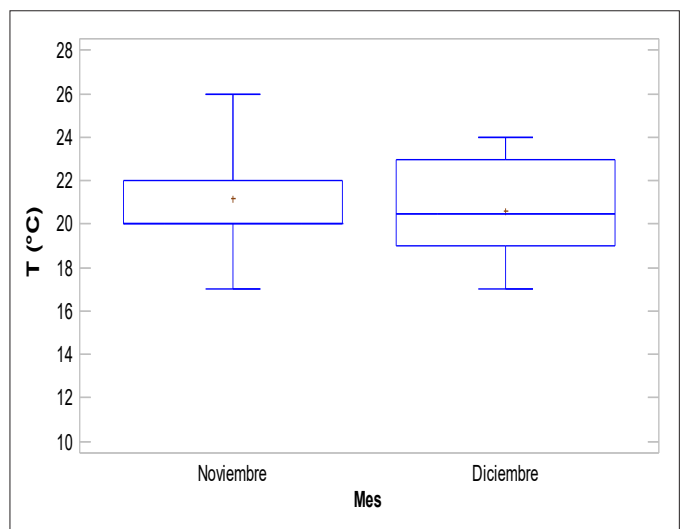

Figura 35. Diferencias significativas de la temperatura del suelo durante noviembre y diciembre de 2019 , tomando como referencia $\mathrm{p}<0,05$ según Mann Whitney.

Humedad del suelo en relación con los cultivos La humedad promedio del suelo para el cultivo de tomate estuvo en $17 \pm 1,99 \%$ y para el suelo del fréjol en $22,62 \pm 6,88 \%$ (Fig. 44). Sí existe una diferencia estadísticamente significativa $(\mathrm{W}=237,0$; $\mathrm{p}=0,0184119$ ) en comparación de la humedad del suelo con los cultivos (Fig. 45).

\section{Resultados de la textura y color del suelo}

La textura del suelo en los sitios de muestreo presentó características de tipo franco, es decir que poseen proporciones idóneas de arcilla, arena y limo para la producción de cultivos; en cuanto al color del suelo, este presentó tonalidades similares desde suelos oscuros hasta el negro parduzco (Tabla 7).

Comparación de cadmio entre los cultivos de tomate y fréjol durante noviembre y diciembre de 2019 en el cantón Pallatanga

La concentración promedio de Cd presente en los suelos agrícolas de tomate y fréjol del cantón Pallatanga varían en función a los sistemas de cultivo, siendo durante noviembre de 2019 donde se registró $0,83 \pm 0,07 \mathrm{mg} / \mathrm{kg}$ para el cultivo de tomate; mientras

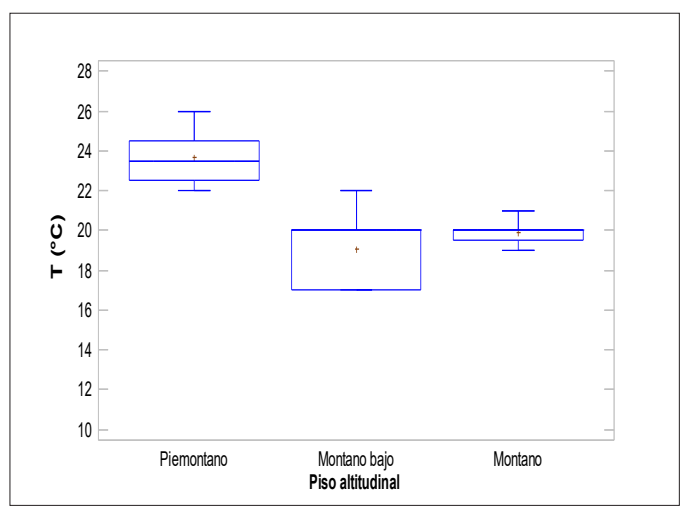

Figura 37. Diferencias significativas entre los pisos altitudinales y temperatura del suelo, tomando como referencia $p<0,05$ según Kruskal Wallis. 
Rev. Cient. Cien. Nat. Ambien. 14(1):151-172 Junio 2020
Pilco \& García - Determinación de cadmio en suelos agrícolas del cantón Pallatanga, provincia de Chimborazo, Ecuador

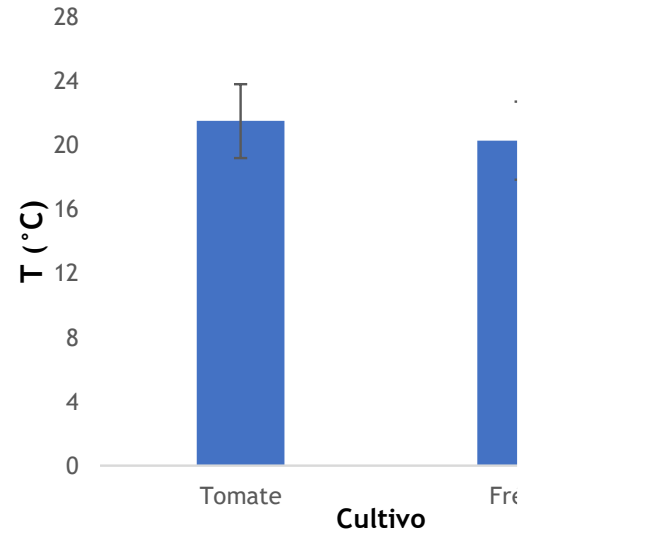

Figura 38. Temperatura del suelo de los cultivos de tomate y fréjol en el cantón Pallatanga. Las barras representan el promedio \pm error estándar.

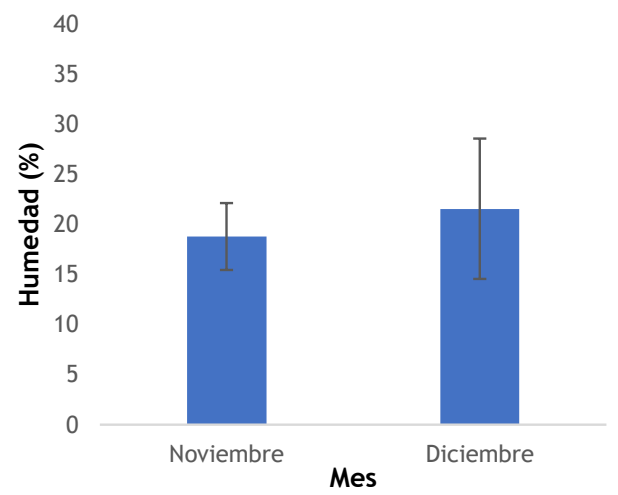

Figura 40. Humedad del suelo durante noviembre y diciembre de 2019 en el cantón Pallatanga. Las barras representan el promedio \pm error estándar.

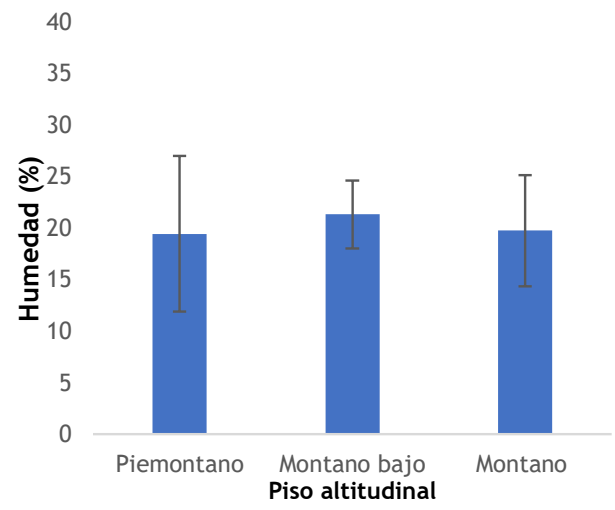

Figura 42. Humedad del suelo en los diferentes pisos altitudinales en el cantón Pallatanga. Las barras representan las medianas \pm error estándar.

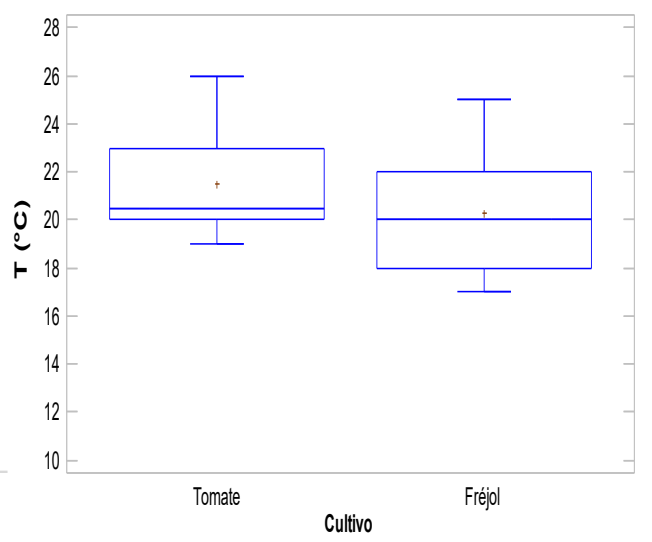

Figura 39. Diferencias significativas de la temperatura del suelo de los cultivos de tomate y fréjol, tomando como referencia $\mathrm{p}<0,05$ según Mann Whitney.

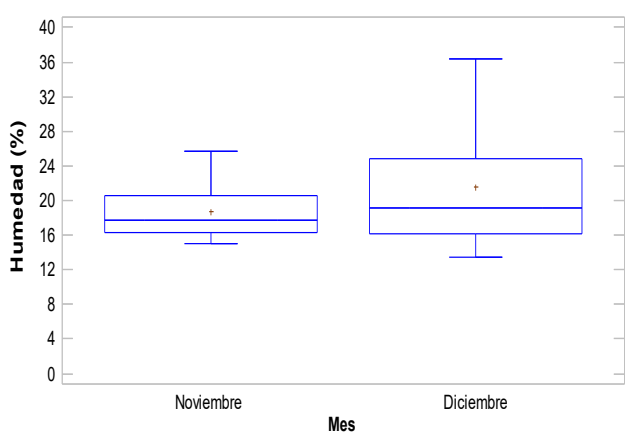

Figura 41. Diferencias significativas de la humedad del suelo durante noviembre y diciembre de 2019, tomando como referencia $\mathrm{p}<0,05$ según Mann Whitney.

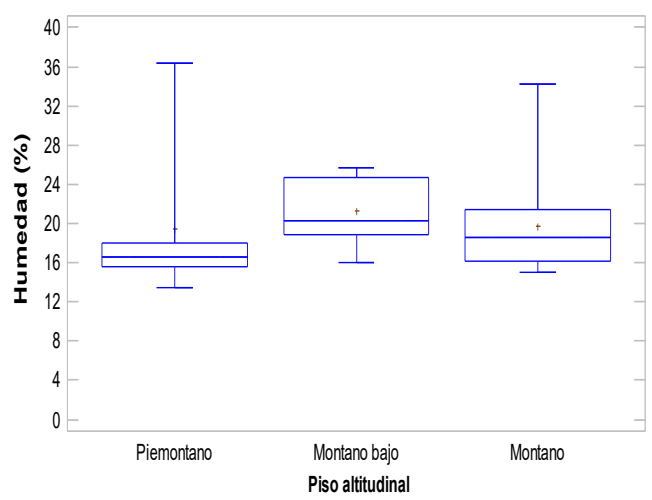

Figura 43. Diferencias significativas entre los pisos altitudinales y humedad del suelo, tomando como referencia $p<0,05$ según Kruskal Wallis. 
Rev. Cient. Cien. Nat. Ambien. 14(1):151-172 Junio 2020
Pilco \& García - Determinación de cadmio en suelos agrícolas del cantón Pallatanga, provincia de Chimborazo, Ecuador

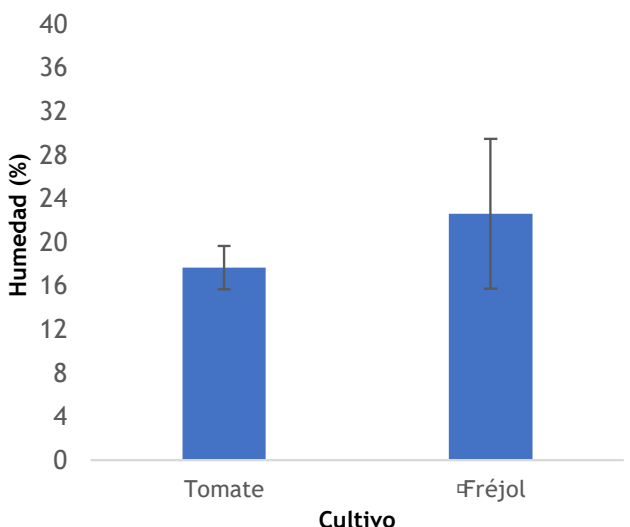

Figura 44. Humedad del suelo de los cultivos de tomate y fréjol en el cantón Pallatanga. Las barras representan el promedio \pm error estándar.

que en el cultivo del fréjol fue $0,70 \pm 0,15 \mathrm{mg} / \mathrm{kg}$ (Fig. 46). Cabe destacar que para ambos cultivos el valor máximo de concentración fue $0.9 \mathrm{mg} / \mathrm{kg} \mathrm{Cd}$; sin embargo, existe una diferencia estadísticamente significativa ( $W=18,5 ; \quad p=0,0492229) \quad$ entre la concentración de $\mathrm{Cd}$ con los cultivos estudiados durante noviembre de 2019 (Fig. 47).

En tanto, para diciembre la concentración promedio de $\mathrm{Cd}$ en los suelos agrícolas de fréjol fue superior con respecto a noviembre. El cultivo de tomate mostró un valor de $0,79 \pm 0,08 \mathrm{mg} / \mathrm{kg}$; mientras que en el de fréjol fue de $0,82 \pm 0,13 \mathrm{mg} / \mathrm{kg}$ (Fig. 46). Sin embargo, el valor máximo de $\mathrm{Cd}$ se registró en los suelos del cultivo de fréjol de $1 \mathrm{mg} / \mathrm{kg}$. A diferencia de noviembre, no se registró una diferencia estadísticamente significativa $(\mathrm{W}=44,0 ; p=0,78017)$ entre la concentración de Cd con los cultivos de tomate y fréjol (Fig. 48).

Comparación de cadmio entre los cultivos de tomate y fréjol que se localizan dentro de tres pisos altitudinales del cantón Pallatanga

La concentración promedio de $\mathrm{Cd}$ en los suelos agrícolas entre los pisos altitudinales mostró varia-

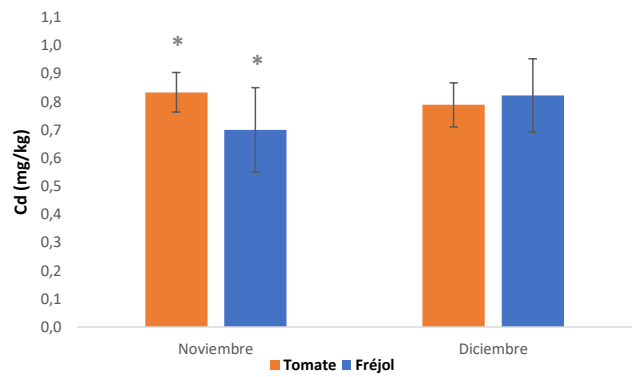

Figura 46. Comparación de la concentración promedio de $\mathrm{Cd}$ en los suelos entre el cultivo de tomate y fréjol durante noviembre y diciembre de 2019 en el cantón Pallatanga. Las barras representan el promedio \pm error estándar: * muestra la existencia de diferencias significas.

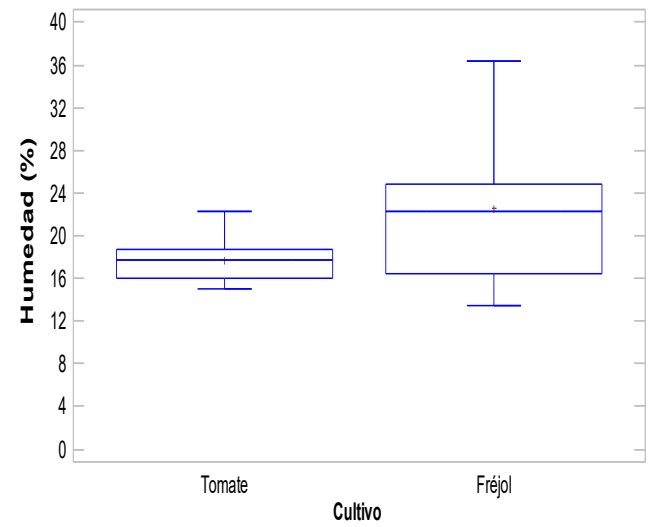

Figura 45. Diferencias significativas de la humedad del suelo de los cultivos de tomate y fréjol, tomando como referencia $\mathrm{p}<0,05$ según Mann Whitney.

ciones entre los cultivos de tomate y fréjol. En el primer piso altitudinal se cuantificó un promedio de $0,78 \pm 0,04 \mathrm{mg} / \mathrm{kg} \mathrm{Cd}$ para el cultivo de tomate y de $0,63 \pm 0,12 \mathrm{mg} / \mathrm{kg}$ Cd para el fréjol (Fig. 49). Hay que destacar el valor máximo de Cd para ambos cultivos el cual fue de $0,8 \mathrm{mg} / \mathrm{kg}$; valores que presentaron una diferencia estadísticamente significativa $(\mathrm{W}=4,5 ; \mathrm{p}=0,0247978)$ entre la concentración de $\mathrm{Cd}$ y los suelos de los cultivos localizados en el primer piso altitudinal (Fig. 50).

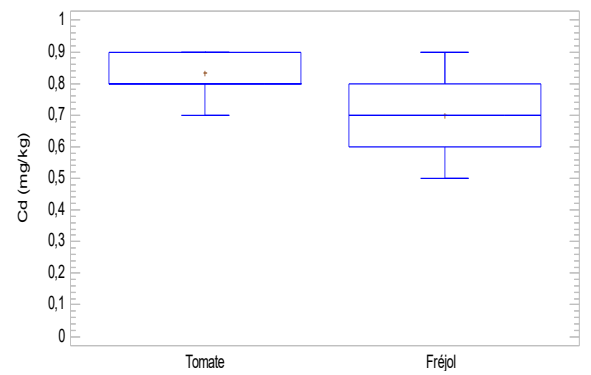

Figura 47. Diferencias significativas de los cultivos con la concentración de Cd para el mes de noviembre de 2019 tomando como referencia $\mathrm{p}<0,05$ según Mann Whitney.

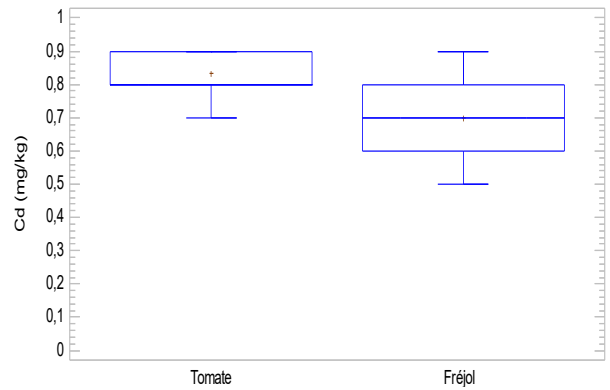

Figura 48. Diferencias significativas de los cultivos con la concentración de Cd para el mes de diciembre de 2019, tomando como referencia $\mathrm{p}<0,05$ según Mann Whitney. 
Tabla 7. Resultados de la textura y color de los suelos agrícolas.

\begin{tabular}{ccccc}
\hline \multirow{2}{*}{ Piso altitudinal } & Cultivo & Abreviatura & Textura & Color \\
\hline \multirow{2}{*}{ Piemontano } & Tomate & PMT & Franco arcilloso limoso & Oscuro \\
\cline { 2 - 5 } & Fréjol & PMF & Franco limoso & Marrón grisáceo \\
\hline \multirow{2}{*}{ Montano bajo } & Tomate & MBT & Franco arcilloso limoso & Negro parduzco \\
\cline { 2 - 5 } & Fréjol & MBF & Franco arenoso & Oscuro \\
\hline \multirow{2}{*}{ Montano } & Tomate & MT & Franco arenoso & Marrón grisáceo \\
\cline { 2 - 4 } & Fréjol & MF & Franco limoso & Negro parduzco
\end{tabular}

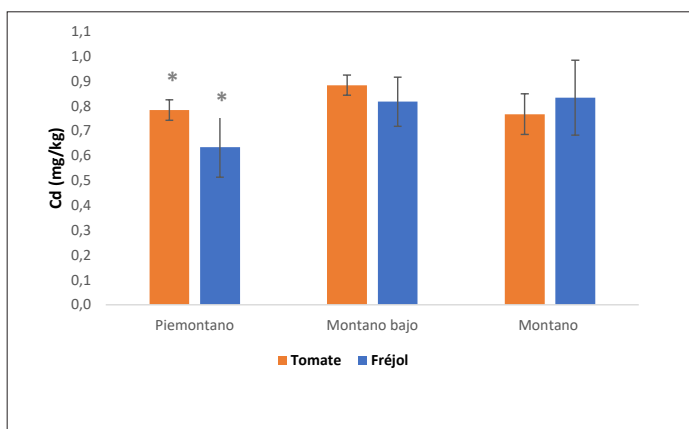

Figura 49. Comparación de la concentración promedio de Cd en los suelos entre los cultivos de tomate y fréjol localizados en tres pisos altitudinales en el cantón Pallatanga. Las barras representan el promedio \pm error estándar: * muestra la existencia de diferencias significas.

Para el piso altitudinal montano bajo al igual que en el primer piso, se registró la mayor concentración de Cd en el cultivo de tomate con 0,88 $\pm 0,04 \mathrm{mg} / \mathrm{kg}$; mientras

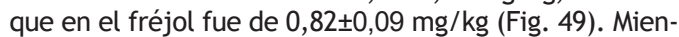
tras que la concentración máxima fue de $0,9 \mathrm{mg} / \mathrm{kg}$ para ambos cultivos, valores que no generaron diferencias estadísticamente significativas ( $\mathrm{W}=11,0 ; \mathrm{p}=0,213258$ ) para la concentración de $\mathrm{Cd}$ y los suelos agrícolas localizados en el piso altitudinal montano bajo (Fig. 51).

Por otra parte, en el piso altitudinal montano se presentó promedios de $\mathrm{Cd}$ diferentes a los pisos piemontano y montano bajo, debido a que el cultivo de fréjol registró concentraciones de $0,83 \pm 0,15 \mathrm{mg} / \mathrm{kg} \mathrm{Cd}$, y en menor presencia en el cultivo de tomate con $0,77 \pm 0,08 \mathrm{mg} / \mathrm{kg}$ (Fig. 49). En donde la máxima concentración del metal en los suelos fue en el cultivo de fréjol con $1 \mathrm{mg} / \mathrm{kg}$, y no se encontró una diferencia estadísticamente significativa ( $\mathrm{W}=22,0 ; \mathrm{p}=0,547267$ ) entre la concentración de $\mathrm{Cd}$ y los cultivos localizados en el piso altitudinal montano (Fig. 52).

Correlación de la concentración de cadmio presente en el suelo del cultivo de tomate con las características fisicoquímicas

La varianza acumulada de valores de las variables en

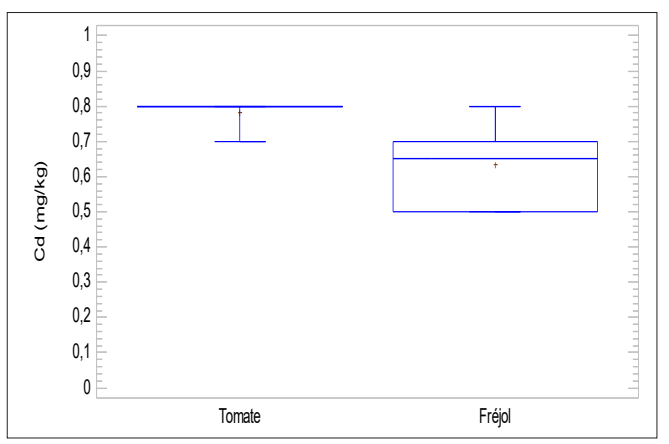

Figura 50. Diferencias significativas de la concentración de $\mathrm{Cd}$ en cultivos de tomate y fréjol localizados en el piso Piemontano, tomando como referencia $\mathrm{p}<0,05$ según Mann Whitney.

los tres primeros componentes del ACP en los suelos agrícolas del cultivo de tomate mostró un $84 \%$ del total (Tabla 8). En el primer componente se demostró la existencia de una correlación positiva y moderada $(=0,45)$ con el $\mathrm{pH}$, débil $(=0,27)$ con la Humedad y muy débil $(=0,08)$ con la materia orgánica; mientras que la temperatura y la conductividad eléctrica obtuvieron una correlación negativa y moderada con rangos de entre $(=-0,53)$ y $(=-0,44)$, respectivamente. En tanto en el segundo componente la variable de interés (Cd) presentó una correlación negativa con todas las variables ambientales en el cultivo de tomate (Fig. 48).

Correlación de la concentración de cadmio presente en el suelo del cultivo de fréjol con las características fisicoquímicas

La varianza acumulada de valores de las variables en los tres primeros componentes del ACP en los suelos agrícolas del cultivo de fréjol mostró un $81 \%$ del total (Tabla 9). En el primer componente se demostró la existencia de una correlación positiva y moderada $(=0,52)$ con la materia orgánica, débil $(=0,35)$ con el $\mathrm{pH}$ y muy débil $(=0,08)$ con la humedad; mientras que la temperatura y la conductividad eléctrica tienen una relación inversa con intensidades entre moderada (=- 
Rev. Cient. Cien. Nat. Ambien. 14(1):151-172 Junio 2020
Pilco \& García - Determinación de cadmio en suelos agrícolas del cantón Pallatanga, provincia de Chimborazo, Ecuador

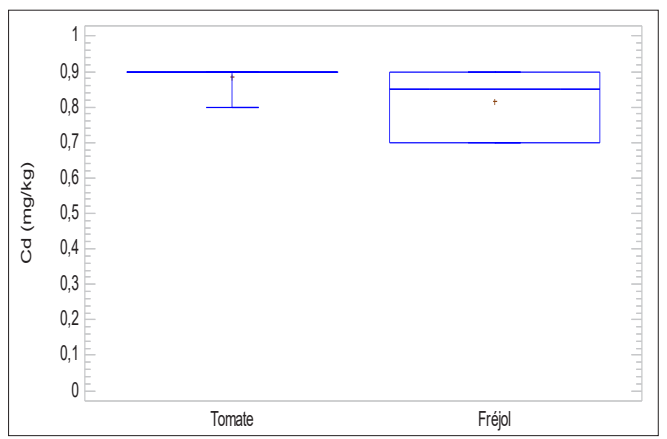

Figura 51. Diferencias significativas de la concentración de $\mathrm{Cd}$ en los cultivos de tomate y fréjol localizados en el piso montano bajo, tomando como referencia $\mathrm{p}<0,05$ según Mann Whitney.

Tabla 8. Porcentaje de varianza explicada por los tres primeros componentes del ACP aplicados a las variables medidas en los suelos del cultivo de tomate en el cantón Pallatanga.

\begin{tabular}{cccc}
\hline Componente & Valor & $\begin{array}{c}\text { Porcentaje } \\
\text { de varianza }\end{array}$ & $\begin{array}{c}\text { Porcentaje } \\
\text { acumulado }\end{array}$ \\
\hline 1 & 2,77 & 46,14 & 46,14 \\
\hline 2 & 1,55 & 25,91 & 72,05 \\
\hline 3 & 0,75 & 12,43 & $\mathbf{8 4 , 4 8}$ \\
\hline
\end{tabular}

Tabla 9. Porcentaje de varianza explicada por los tres primeros componentes del ACP aplicados a las variables medidas en los suelos del cultivo de fréjol en el cantón Pallatanga.

\begin{tabular}{cccc}
\hline Componente & Valor & $\begin{array}{c}\text { Porcentaje } \\
\text { de varianza }\end{array}$ & $\begin{array}{c}\text { Porcentaje } \\
\text { acumulado }\end{array}$ \\
\hline 1 & 2,66 & 44,27 & 44,27 \\
\hline 2 & 1,22 & 20,28 & 64,55 \\
\hline 3 & 0,97 & 16,09 & $\mathbf{8 0 , 6 4}$ \\
\hline
\end{tabular}

$0,55)$ y débil $(=-0,33)$, respectivamente en el primer componente. En tanto en el segundo componente sólo la variable temperatura mostró una correlación negativa y muy débil $(=-0,04)$, con la concentración de cadmio en el cultivo de fréjol (Fig. 49).

\section{Discusión}

El cadmio (Cd) es un metal tóxico que se incorpora en la dieta humana a través de los alimentos, y estos a su vez de los suelos al encontrarse biodisponible; de forma general el cadmio encontrado en los suelos agrícolas del cantón Pallatanga, sobrepasaron el límite máximo permisible nacional para los tres pisos altitudinales, y del mismo modo para los cultivos de tomate y fréjol estudiados. Además, las características fisicoquímicas

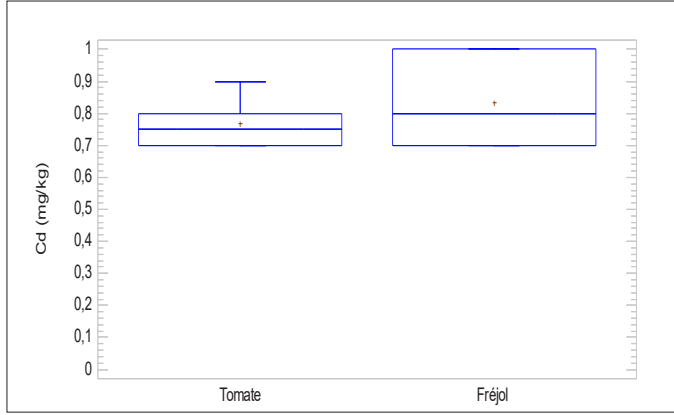

Figura 52. Diferencias significativas de la concentración de $\mathrm{Cd}$ en los cultivos de tomate y fréjol localizados en el piso montano, tomando como referencia $\mathrm{p}<0,05$ según Mann Whitney.

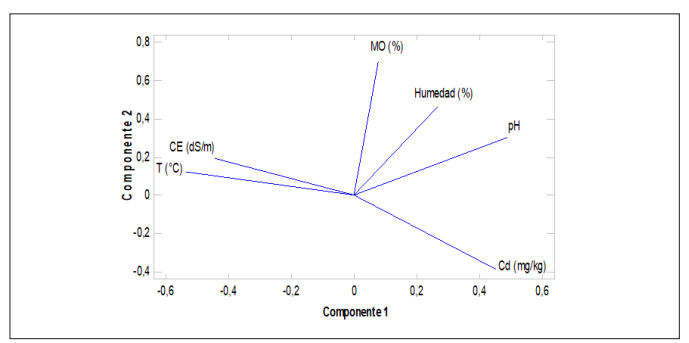

Figura 53. Análisis de componentes principales entre las variables estudiadas del cultivo de tomate: concentración de $\mathrm{Cd}, \mathrm{pH}$, materia orgánica, conductividad eléctrica, humedad y temperatura del suelo.

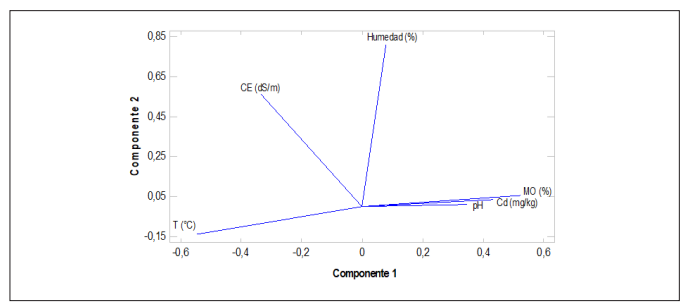

Figura 54. Análisis de componentes principales entre las variables estudiadas del cultivo de fréjol: concentración de $\mathrm{Cd}, \mathrm{pH}$, materia orgánica, conductividad eléctrica, humedad y temperatura del suelo.

de los suelos en cuestión resultaron ser propicias para la retención del metal cadmio.

En este estudio se demuestra que la variabilidad del $\mathrm{Cd}$ en los suelos agrícolas entre los meses de noviembre y diciembre de 2019 difiere con los tipos de cultivo y pisos altitudinales, con valores entre $0,5 \mathrm{mg} / \mathrm{kg}$ y 1,0 $\mathrm{mg} / \mathrm{kg}$. El $94 \%$ de las muestras superan el LMP nacional (TULSMA), el cual establece como valor límite $0,5 \mathrm{mg} /$ $\mathrm{kg}$ de $\mathrm{Cd}$, mientras que el $33 \%$ de los mismos superaron las directrices holandesas $(0,80 \mathrm{mg} / \mathrm{kg})$.

Estos datos se asemejan a los reportados por Chavez et al. (2015), cuyas muestras fueron tomadas a la misma profundidad $(0-15 \mathrm{~cm})$, en donde obtuvo valores que fluctuaron entre $0,66 \mathrm{mg} / \mathrm{kg}$ y $2,59 \mathrm{mg} / \mathrm{kg}$ de cadmio. Es muy probable que los valores elevados de 
cadmio encontrados en los suelos agrícolas del cantón Pallatanga se deban principalmente al inadecuado uso de fertilizantes químicos, siendo menos posible que este metal ingrese a los suelos a través de la irrigación debido a que no se encontraron fuentes de contaminación que pudieran afectar el agua de riego (Sheppard et al., 2009; Alloway \& Steinnes, 1999) including soil, but agriculture uses some materials that have increased TE concentrations. Some TEs (e.g., Cu, $\mathrm{Se}$, and $\mathrm{Zn}$, esto es respaldado por estudios previos realizados (Qian et al., 2016; Muñoz et al., 2017), este último realizado en la provincia del Guayas, quienes identificaron a los fertilizantes químicos como fuentes de contaminación de cadmio en los suelos agrícolas, debido a que encontraron valores de $56 \mathrm{mg} / \mathrm{kg}$ y 41,30 $\mathrm{mg} / \mathrm{kg}$, respectivamente.

La concentración de $\mathrm{Cd}$ en los pisos altitudinales presentó mayor acumulación del metal en el montano bajo $(0,85 \pm 0,08 \mathrm{mg} / \mathrm{kg})$, seguido de los pisos montano $(0,80 \pm 0,12 \mathrm{mg} / \mathrm{kg})$ y piemontano $(0,71 \pm 0,12)$, respectivamente. Estos valores difieren a un estudio previo (Felicita, 2018), que registró concentraciones por debajo de $0,2 \mathrm{mg} / \mathrm{kg}$ en suelos de la cuenca baja del río Guano. Probablemente esta diferencia se debe a que en esta zona no se evidenció una actividad agrícola extensiva. Cabe indicar que las concentraciones de $\mathrm{Cd}$ halladas en los pisos altitudinales sugieren que la movilidad de este metal está relacionada con las actividades antropogénicas y las características fisicoquímicas de los suelos al no inferir la altitud del terreno en la concentración del metal, dato que ha sido afirmado (Alloway, 2013).

Respecto a los cultivos, se determinó una mayor acumulación del metal en el cultivo de tomate $(0.81 \pm 0,08)$, a diferencia del fréjol $(0,76 \pm 0,15$ $\mathrm{mg} / \mathrm{kg}$ ). Estos resultados son comparables con otros estudios realizados (Escobar, 2016), cuyo valor medio para los suelos de tomate fue de $0,56 \mathrm{mg} / \mathrm{kg}$; por el contrario, la investigación realizada por Meers et al. (2006)awareness has risen that the total soil content of pollutants by itself does not suffice to fully assess the potential ecotoxicological risks involved. Chemical analysis will require to be complemented with biological assays in a multidisciplinary approach towards site specific ecological risk assessment (SSERA en suelos de fréjol, determinó un valor de 7,61 $\mathrm{mg} / \mathrm{kg}$ de cadmio. La mayor concentración del cadmio en los suelos de tomate podría deberse a una menor biodisponibilidad de este metal, lo que provocaría una mayor acumulación en el suelo y no en la planta del tomate (Singh \& McLaughlin, 1999).

Con respecto a las características fisicoquímicas de los suelos agrícolas del cantón Pallatanga, la variable ambiental $\mathrm{pH}$ obtuvo valores que fluctuaron entre 4,0 y 7,0 , en donde el $69 \%$ de los suelos estudiados resultaron ser moderadamente ácidos según la Secretaría de Medio Ambiente y Recursos Naturales (2002), detectando un mayor grado de acidez en el piso piemontano, mientras que para los sistemas de cultivo el pH no mostró diferencias significativas $(p>0,05)$. La materia orgánica $(M O)$ por su parte presentó variaciones del $5,20 \%$ y $11,35 \%$, siendo en un $64 \%$ de los suelos considerados como medios en MO, en donde se presentó una mayor acumulación de MO en el piso montano bajo, y en el cultivo de fréjol. Según Bernardo et al. (2019), las condiciones ácidas aumentan la fracción de Cd soluble () a consecuencia de la disolución de los carbonatos y sulfatos lo que incrementa su solubilidad; y los porcentajes mayores al $5 \%$ de $\mathrm{MO}$ presentan un nivel relativamente bajo de captura de Cd por las plantas, a consecuencia de la alta capacidad de las moléculas orgánicas para formar complejos (Chicón, 2006). Una forma de aumentar el pH del suelo es a través del encalado, lo que disminuye la disponibilidad del cadmio para las plantas (Puschenreiter, Horak, Friesl-Hanl, \& Hartl, 2005). Otra alternativa para mejorar las condiciones de los suelos es la incorporación de enmiendas orgánicas como el compost, estiércol de granja, y los biosólidos, debido a su alto contenido de materia orgánica, fósforo, y hierro. Según Krishnamurti, Huang, \& Kozak (1999), la biodisponibilidad del cadmio en suelos con $\mathrm{pH}$ de 6,1 y 7,8 , con contenidos de materia orgánica de $3,9 \%$ y $3 \%$, disminuye al incrementar la cantidad de fósforo en el suelo.

Hay que recalcar que todos los suelos de esta investigación presentaron texturas francas según las tablas de Munsell (2009), es decir, presentaron proporciones idóneas de arcilla, arena y limo, con efectos despreciables de salinidad $(<1,0 \mathrm{dS} / \mathrm{m})$. Por lo que es conocido, este tipo de suelos que contienen arcilla tienden a adsorber al metal Cd (Bernardo et al., 2019); pero a su vez el aumento de la salinidad puede incrementar su movilidad (Galán \& Romero, 2008)hidrosfera y biosfe-ra sobre la superficie de la geosfera. Ocupa la interfase entre la geosfera y los demás sistemas, en la llamada Zona Crítica (National Research Council, 2001; Brantley et.al. 2007.

Los resultados obtenidos en la presente investigación muestran que las concentraciones de $\mathrm{Cd}$ no dependen de la altitud del terreno; sino más bien de la intensidad de actividades agrícolas que se presentan en la zona. Por lo antes expuesto, se asume que los suelos agrícolas del piso altitudinal piemontano no presentan características fisicoquímicas adecuadas para la retención del $\mathrm{Cd}$, especialmente con la variable $\mathrm{pH}$ y $\mathrm{MO}$, posiblemente este metal se encuentre con una alta movilidad y biodisponibilidad en el suelo independientemente de los sistemas de cultivo, lo que podría explicarse en parte porque existe una menor acumulación de $\mathrm{Cd}$, a pesar de que existe una alta actividad agrícola en este piso altitudinal; mientras que en el montano bajo y montano presentaron condiciones idóneas para la retención del metal, debido a que ahí se encontraron las concentraciones más elevadas de Cd específicamente en el piso montano bajo, así como en los suelos del cultivo de tomate.

Finalmente, en la correlación de las variables ambientales con la concentración de $\mathrm{Cd}$ que se 
encontraron en los sistemas de cultivo, se observó claramente que la variable $\mathrm{pH}$ y $\mathrm{MO}$, estuvieron asociados positivamente a la presencia del metal, lo que corrobora lo antes planteado especialmente esto se pudo observar en el cultivo de fréjol.

\section{Conclusiones}

De acuerdo con las observaciones de campo y la caracterización fisicoquímica de los suelos agrícolas del cantón Pallatanga, se puede afirmar que estos son moderadamente ácidos, con tonalidades oscuras debido a su naturaleza volcánica y principalmente a la presencia considerable de materia orgánica, presentando texturas francas propicias para la producción de cultivos y la retención del cadmio.

Las concentraciones medias de cadmio registradas entre noviembre y diciembre del 2019 sobrepasaron la normativa nacional (TULSMA) en los tres pisos altitudinales, y de igual forma para los dos sistemas de cultivos estudiados; mientras que para las directrices holandesas solamente el piso altitudinal montano bajo y el cultivo de tomate (Solanum lycopersicum) estuvieron fuera de normativa.

El análisis de componentes principales mostró una correlación moderada para la materia orgánica, y débil para el $\mathrm{pH}$ para ambos cultivos, siendo más significativos en el cultivo de fréjol (Phaseolus vulgaris).

\section{Recomendaciones}

Preparar los terrenos antes de cada siembra, siendo fundamental la estabilización química del suelo ya sea con el encalado o la incorporación de enmiendas orgánicas, de tal forma que el cadmio no se encuentre biodisponible para los cultivos.

- Realizar análisis fisicoquímicos del terreno antes de la aplicación de fertilizantes químicos, para determinar la cantidad exacta que se debe emplear, ya que además de representar un desperdicio del producto que a su vez aumenta el costo de producción, genera un impacto adverso en el suelo.

- Para aplicar medidas más efectivas en el control de los suelos del cantón Pallatanga, se recomienda ampliar el campo de análisis de los metales pesados en los cultivos, determinar su origen y el impacto que generan en los suelos agrícolas.

\section{Bibliografía}

Alloway, B.J. 2013. Sources of heavy metals and metalloids in soils. In: B. Alloway, Heavy Metals in Soils, Trace metals and Metalloids in soils and their Bioavailability. Springer, Dordrecht (2013), pp. 11-50.

Alloway, B.J., y Steinnes, E. 1999. Anthropogenic Additions of Cadmium to Soils. In Cadmium in Soils and Plants. 85: 97-123.

Añazco, K. 2019. Efectos del cadmio sobre la germinación y crecimiento de Lens culinaris Medik. (Lenteja), Oryza Sativa L. (Arroz) y Phaseolus vulgaris L. (Fréjol). Tesis de grado previo a la obtención de Ingeniero Ambiental, Facultad de Ciencias Naturales. Universidad de Guayaquil. Recuperado de http://repositorio.ug.edu.ec/handle/ redug/39572

Bernardo, A., Fernández, C., Rocha, D., Chepen, K., \& Rivas, N. 2019. Procesos químicos y bioquímicos del cromo y del cadmio en el suelo. https://doi.org/10.13140/ RG.2.2.31087.18080

Bonomelli, C., Bonilla, C., y Valenzuela, A. 2003. Efecto de la fertilización fosforada sobre el contenido de cadmio en cuatro suelos de Chile. Pesquisa Agropecuaria Brasileira, 38(10): 1179-1186.

Boyer, J., Fourqurean, J., y Jones, R. 1997. Spatial Characterization of Water Quality in Florida Bay and Whitewater Bay by Multivariate Analyses: Zones of Similar Influence. Estuaries and Coasts, 20: 743-758.

Cargua, J.E. 2010. Determinación de las Formas de Cu, Cd, $\mathrm{Ni}, \mathrm{Pb}$ y $\mathrm{Zn}$ y su disponibilidad en suelos agrícolas del litoral ecuatoriano. Tesis de grado previo a la obtención de título de Ingeniera Agropecuaria: Mención en Producción Pecuaria, Universidad Tecnológica Equinoccial.

Castro, C. 2018. Tabla Munsell Teoría y Práctica y Manejo de Tabla de colores Munsell y régimen de Humedad. https: / / doi.org/10.13140/RG.2.2.19472.05125

Chavez, E., He, Z.L., Stoffella, P.J., Mylavarapu, R.S., Li, Y.C., Moyano, B., y Baligar, V.C. 2015. Concentration of cadmium in cacao beans and its relationship with soil cadmium in southern Ecuador. Science of the Total Environment, 533: 205-214.

Chicón, L. 2006. Especiación de metales pesados en lodos de aguas residuales de origen urbano y aplicación de lodos digeridos como mejoradores de suelos Trabajo de Investigación del Programa de Doctorado en Ingeniería Ambiental, Universidad de Málaga. Recuperado de https: / / www. pilcomayo.net/media/uploads/biblioteca/ libro_902_MA-271.pdf

Cuadras C. 1983. Analisis de la varianza y covarianza en problemas de probabilidad y estadistica. Edit. Reverte, S. A. Barcelona, España. 22 pp.

Danielyan, K.E., y Chailyan, S.G. 2019. Heavy Metals. Biomedical Journal of Scientific \& Technical Research, 21(5): 16165-16169.

Escobar, S. 2016. Determinación de la presencia de plomo y cadmio en frutilla (Fragaria ananassa) y tomate (Solanum lycopersicum) en el Quinche. Trabajo de grado previo a la obtención de título de Ingeniero Agrónomo, Facultd de Ciencias Agrícolas, Universidad Central del Ecuador. Recuperado de http://www.dspace.uce.edu.ec/ handle/25000/10068

Evans, J. 1996. Straightforward statistics for the behavioral sciences. Thomson Brooks/Cole Publishing Co.

FAO y WHO. 2019. Codex Alimentarius Commission - Procedural Manual twenty-seventh edition. Rome. 254 pp.

Felicita, O.M. 2018. Estudio de presencia de contaminantes (metales pesados y agrotóxicos) en agua, suelo y alimentos en los cantones Riobamba y Guano de la provincia de Chimborazo y su posible relación con el espectro Autista. Informe final de Investigación, Universidad Andina Simón Bolivar, sede Ecuador, 56 p. https://repositorio.uasb.edu. 
ec/bitstream/10644/6711/1/PI-2018-15-Felicita-Estudio. pdf

Galán, E., y Romero, A. 2008. Contaminación de Suelos por Metales Pesados. Macla 10: 48-60. Recuperado de http:// www.ehu.eus/sem/macla_pdf/macla10/Macla10_48.pdf

Gobierno Autónomo Descentralizado Municipal del Cantón Pallatanga. 2018. Plan de ordenamiento territorial del gobierno autónomo descentralizado del cantón Pallatanga reforma 2018. Recuperado de https://pallatanga.gob.ec/ index.php/gad/planes-de-gobierno

Grant, C.A., Buckley, W.T., Bailey, L.D., y Selles, F. 1998. Cadmium accumulation in crops. Canadian Journal of Plant Sciences, 78: 1-17. https://doi.org/10.4141/P96-100

Kabata-Pendias, A. 2010. Trace elements in soils and plants: Fourth edition. CRC press, Boca Ratón, Fl. https://doi. org/10.1201/b10158

Kobayashi, J. 1978. Pollution by cadmium and the itai-itai disease in Japan. In: Oehme F.W. ed. Toxicity of Heavy Metals in the Environment, New York, Marcel Dekker, pp. 199-260.

Krishnamurti, G., Huang, P., \& Kozak, L. 1999. Sorption and Desorption Kinetics of Cadmium From Soils: Influence of Phosphate. Soil Science, 164: 888-898.

Marrugo-Negrete, J., Pinedo-Hernández, J., y Díez, S. 2017. Assessment of heavy metal pollution, spatial distribution and origin in agricultural soils along the Sinú River Basin, Colombia. Environmental Research, 154: 380-388.

Meers, E., Ruttens, A., Geebelen, W., Vangronsveld, J., Samson, R., Vanbroekhoven, K., Vandegehuchte, M., Diels, L., y Tack, F.M.G. 2006. Potential use of the plant antioxidant network for environmental exposure assessment of heavy metals in soils. Environmental Monitoring and Assessment, 120(1-3): 243-267.

Mendoza, R. B., y Espinoza, A. 2017. Guía Técnica para Muestreo de Suelos. Recuperado de http://repositorio. una.edu.ni/3613/1/P33M539.pdf

Ministerio de Ambiente del Ecuador. 2012. Metodología para la Representación Cartográfica de los Ecosistemas del Ecuador Continental. Recuperado de https://www.ambiente.gob. ec/wp-content/uploads/downloads/2012/09/Documento_ Metodolog+ia_28_05_2012_v2_1.pdf

Ministerio del Ambiente. 2015. Acuerdo no. 061 reforma del libro VI del Texto Unificado de Legislación Secundaria. Recuperado de http://suia.ambiente.gob. ec/ documents/10179/185880/ACUERDO+061+REFOR $M A+L I B R O+V I+T U L S M A+-+R \cdot 0 \cdot 316+04+D E+M A Y O+2015$. pdf /3c02e9cb-0074-4fb0-afbe-0626370fa108?version=1.0

Miyazawa, M., Pavan, M.A., Oliveira, E.L. de, Ionashiro, M., y Silva, A.K. 2000. Gravimetric determination of soil organic matter. Brazilian Archives of Biology and Technology, 43(5): 475-478.

Muñoz, J. D. 2017. Determinación de Cadmio en fertilizantes, plantas de Oryza sativa L. y suelos de la provincia del Guayas: Propuesta de saneamiento. Universidad de Guayaquil.

Munsell Color. 2009. Munsell Soil Color Charts: With Genuine Munsell Color Chips (2009th ed.). Recuperado de https: / / books.google.com.ec/books?id=X8GQxgEACAAJ\&dq=MUNS $\mathrm{ELL}+\mathrm{SOIL}+\mathrm{COLOR}+\mathrm{CHARTS}+2009 \& \mathrm{th}=$ es\&sa $=$ X\&ved=0ahUKE wjMIZ-smdPoAhUESN8KHQIXAzcQ6AEIKjAA

Neelam, K., Vinita, R., y Sibi, J. 2018. Human Health and effects of heavy metals. International Journal of Applied Chemistry, 5(1): 4-5.

Proshad, R., Islam, M., Kormoker, T., Bhuyan, M., Hanif, M., Hossain, N., Roy, R. y Sharma, A. 2019. Contamination of Heavy Metals in Agricultural Soils: Ecological and Health Risk Assessment. SF Journal of Nanochemistry and Nanotechnology, 2(1): 1-13.

Puschenreiter, M., Horak, O., Friesl, W., y Hartl, W. 2005. Low-cost agricultural measures to reduce heavy metal transfer into the food chain - A review. Plant, Soil and Environment, 51: 1-11.
Qian, M., Wu, H., Wang, J., Zhang, H., Zhang, Z., Zhang, Y., Lin, H., y Ma, J. 2016. Occurrence of trace elements and antibiotics in manure-based fertilizers from the Zhejiang Province of China. Science of The Total Environment, 559: 174-181.

Ritchey, E.L., Mcgrath, J.M., y Gehring, D. 2015. Determining Soil Texture by Feel. Agriculture and Natural Resources Publications. 139. Recuperado de https: //uknowledge.uky. edu/anr_reports/139

Rodrigues, L., Reis, A., y Lupino, P. 2016. Heavy metals in agricultural soils: From plants to our daily life. Científica, Jaboticabal, 44(3): 346-361.

Salmanzadeh, M., Balks, M.R., Hartland, A., y Schipper, L.A. 2016. Cadmium accumulation in three contrasting New Zealand soils with the same phosphate fertilizer history. Geoderma Regional, 7(3): 271-278.

Sánchez, G. 2016. Ecotoxicología del cadmio riesgo para la salud de la utilización de suelos ricos en cadmio. Trabajo de fin de grado, Facultad de Farmacia, Universidad Complutense. Recuperado de http://147.96.70.122/Web/ TFG/TFG/Memoria/GARA SANCHEZ BARRON.pdf

Schroeder, H.A., y Balassa, J.J. 1963. Cadmium: Uptake by vegetables from superphosphate in soil. Science, 140(3568): 819-820.

SEMARNAT (Secretaría de Medio Ambiente y Recursos Naturales). 2002. Norma Oficial Mexicana NOM-021SEMARNAT-2000, que establece las especificaciones de fertilidad, salinidad y clasificación de suelos, estudios, muestreo y análisis. Diario Oficial, Segunda Sección. Recuperado de http://biblioteca.semarnat.gob.mx/ janium/Documentos/Ciga/libros2009/D02280n.pdf

Sheppard, S.C., Grant, C. A., Sheppard, M.I., de Jong, R., y Long, J. 2009. Risk Indicator for Agricultural Inputs of Trace Elements to Canadian Soils. Journal of Environmental Quality, 38(3): 919-932.

Sierra, C. 2019. La relación entre contenido de arcilla y la CIC y su influencia en la fertilización. Recuperado del sitio web de El Mercurio: Campo: https://digital.elmercurio. com/2020/03/03/A

Singh, B.R., y McLaughlin, M.J. 1999. Cadmium in Soils and Plants. In: Developments in Plant and Soil Sciences, pp. 257-267. https://doi.org/10.1007/978-94-011-4473-5_10

Singhal, R.L., Merali, Z., y Hrdina, P.D. 1976. Aspects of the biochemical toxicology of cadmium. Federation Proceedings, 35: 75-80.

Sokal, R.R., y Rohlf, F.J. 2002. Introducción a la bioestadística. Ed. Reverté. Barcelona, 380 p.

Sonmez, S., Buyuktas, D., Okturen Asri, F., y Citak, S. 2008. Assessment of different soil to water ratios $(1: 1,1: 2.5,1: 5)$ in soil salinity studies. Geoderma, 144: 361-369.

Tóth, G., Hermann, T., Da Silva, M.R., y Montanarella, L. 2016. Heavy metals in agricultural soils of the European Union with implications for food safety. Environment International, 88: 299-309.

Unión Europea. 2019. Contenidos máximos en metales pesados en productos alimenticios. Recuperado de http: / / plaguicidas.comercio.es/MetalPesa.pdf

Wang, P., Chen, H., Kopittke, P.M., y Zhao, F.J. 2019. Cadmium contamination in agricultural soils of China and the impact on food safety. Environmental Pollution, 249: 1038-1048.

Wildlands School. 2010. Soil Science 3. Measuring Soil Moisture and Organic Content. Recuperado de https:// www.youtube. com/watch? $v=X m p r D M q u 4 z c$

Zar, J. 1996. Biostatistical Analysis. 3era Ed. Edit. Prentice Hall. Upper Saddle River New Jersey, USA. 662 pp. 